$\underline{\text { Review Article }}$

\title{
RISE AND FALL IN SARS-COV-2 GLOBAL PANDEMIC STRAIN RATE-AN OVERVIEW
}

\section{ABDULLAH ANWAR ${ }^{a, b^{*}}$, VISHAL SINGH CHANDELc, SATYENDRA PRATAP SINGHd, SATYA PRAKASH SINGHe, NEDA ANWAR ${ }^{f}$}

aDepartment of Civil and Construction Engineering, National Taiwan University of Science and Technology, 106 Taipei, Taiwan, ROC 'Department of Civil Engineering, Rajkiya Engineering College, Ambedkar Nagar, Uttar Pradesh, 224122, India, 'Department of Applied Science and Humanities, Rajkiya Engineering College, Ambedkar Nagar, Uttar Pradesh, 224122, India, dDepartment of Physics, AIAS, Amity University, Noida, Uttar Pradesh, 201301, India, eDepartment of Pharmacy, Babu Sunder Singh College of Pharmacy, Nigohan, Uttar Pradesh, 226302, India, 'Department of Dental Surgery, Career Institute of Medical Sciences and Hospital, Lucknow, India, 226020 *Email: a.anwar14330@gmail.com

Received: 03 Feb 2021, Revised and Accepted: 12 Apr 2021

\section{ABSTRACT}

After its discovery in Hubei in China in December 2019, the deadly rise of modern coronavirus (COVID-19 or 2019-nCoV) has spread globally. SARSCoV-2 disease COVID-19 has quickly spread worldwide, posing a serious threat to health and the economy. As of 25th January 2021, more than 100 million confirmed cases of 2,165,581 deaths have been reported by WHO and Worldometer. Many of the cases reported are caused by infection from human to human and are the carriers of this lethal coronavirus. Due to its calamitous nature, the whole world was under lockdown restricting all sorts of movements and means of transportation in hampering the countries economic balance. Presently, the world's endeavor to create and develop a safe and effective COVID-19 vaccine is bearing the fruit. A handful of vaccines now have been authorized around the globe and many more remain in the development phase. In addition, social isolation and knowledge of hygiene (facial masks and sanitizers) are potential methods of controlling the further dissemination of global pandemics COVID-19. This research article presents a brief overview of the catastrophic effect caused by COVID-19 disease globally and particularly in different states of India. Additionally, the article also discusses the recent variant of SARS-CoV-2 and its vulnerable impact. Furthermore, the article investigates the currently available vaccines and those in their development phase for the treatment of COVID-19 disease. This investigatory literature may provide comprehensive details on COVID-19 disease from its inception to grow and later fall in its strain rate.

Keywords: Coronavirus, COVID-19, 2019-nCoV, SARS-CoV-2, Wuhan, Vaccines

(c) 2021 The Authors. Published by Innovare Academic Sciences Pvt Ltd. This is an open access article under the CC BY license (https://creativecommons.org/licenses/by/4.0/) DOI: https://dx.doi.org/10.22159/ijap.2021v13i3.40994. Journal homepage: https://innovareacademics.in/journals/index.php/ijap Speedy peer review was done as the subject of the manuscript was related with pandemic

\section{INTRODUCTION}

The world is now in a state of crisis [1]. The latest coronavirus epidemic has easily reached the world population, which came into being in Wuhan, Hubei Province of China, on 31 ${ }^{\text {st }}$ December 2019 [2]. Coronaviruses (CoVs) are extremely large viruses with a single strand RNA genome encompassing a membrane shell [3]. The word 'coronavirus,' in electron microscopy, refers to the appearance of CoV virions in which viral membrane is tucked with projections of glycoprotein spikes(S), which give the appearance of the like corona (Crown in Latin) $[4,5]$. Some virally encoded proteins that make up the surface portion of the virus include $\mathrm{E}$ (envelope protein), $\mathrm{M}$ (membrane protein), and HE (haemagglutinin-esterase protein) (fig. 1) $[5,6]$. In many avian hosts $[7,8]$, as well as in different mammals, CoVs have been reported, including camels, bats, masked palm civets, mice, dogs, and cats. Novel mammalian coronaviruses are now regularly identified[10]. For eg, HKU2-related bat coronavirus caused acute pig diarrhea syndrome fatal in 2018 [11]. CoVs affect upper breathing, respiratory, hepatic, and central nervous system disorders [12]. In

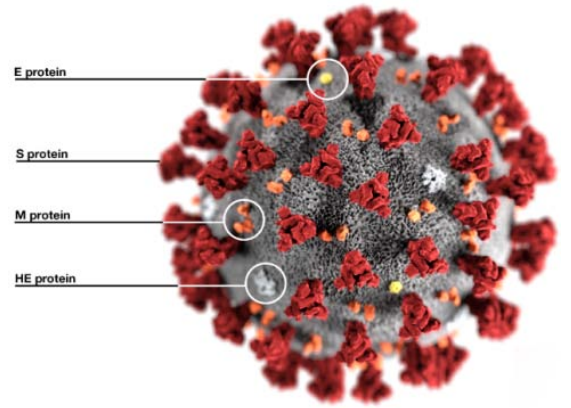

(a)
1965, the first human coronavirus (HCoVs) was isolated from the nasal release of common cold patients named B814 [13]. There are seven multiple CoVs believed to infect humans. The following are $\mathrm{HCoV}-229 \mathrm{E}$ (229E), HCoV-OC43 (OC43), Severe Acute Respiratory Syndrome Coronavirus (SARS-CoV), HCoV-NL63 (NL63), HCoV-HKU1 (HKU1), Middle East Respiratory Syndrome Coronavirus (MERS-CoV), and SARS-CoV-2 [13, 14]. Four of which are 229E, OC43, NL63, and HKU1, responsible for causing mild diseases, and the rest three SARS-CoV, MERS-CoV and SARS-CoV-2 can be even lethal $[15,16]$. The novel beta coronavirus agent SARS-CoV, was found in the Guangdong province of China in 2002-2003 to be a serious acute respiratory disease outbreak [18]. Between 2002 and 2003 there were more than 8000 human infections and 774 deaths in 37 countries [19]. MERS-CoV is a pathogen accountable in 2012 for the continuing emergence of extreme respiratory diseases located in the Middle East, Saudi Arabia [20]. There have been 2494 laboratory-based infection deaths since September 2012 and 858, including 38 in South Korea after single infection [21]. However, the lower air system triggers viral pneumonia, both SARS-CoV and MERS-CoV [10].

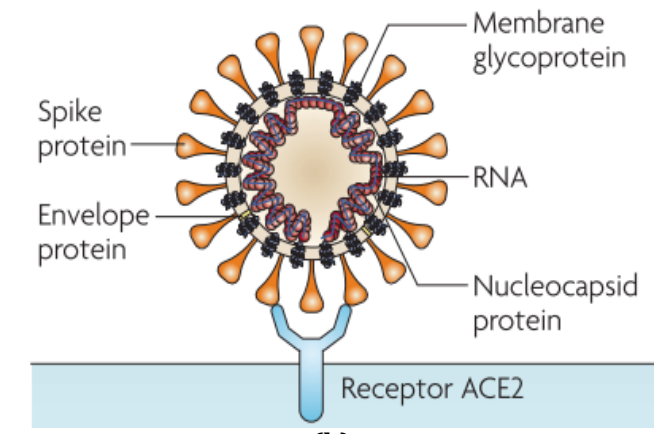

(b)

Fig. 1: (a) Schematic structure of CoVs [6], (b) Cross-sectional view of CoVs structure with a viral receptor on the host cell surface [7] 
On $09^{\text {th }}$ January 2020, the Chinese Centre for Disease Control and Prevention (CCDC) announced the latest human zoonotic coronavirus, SARS-CoV-2 [22]. SARS-CoV-2 does the same thing as SARS-CoV and MERS-CoV but also affects the immune tract, the respiratory system, the kidney, the liver and the center nerves [15]. The Huanan South China Seafood Industry was connected to the initial contaminated cases. Their first case was recorded in China in December 2019 and many scientists suspect that a pandemic is leading the planet to a halt [23]. In China 62 confirmed cases were reviewed by $17^{\text {th }}$ January [24]. Total patients in Wuhan are contaminated until 198 on 19th January. On 20th January, the confirmed cases of 2019-nCoV increased to 282. Four countries, including China (278), Thailand (2 cases), Japan (1 case), and the Republic of Korea have registered these cases (1 case) [25]. The cases have been brought to Thailand, Japan and the Republic of Korea from Wuhan City, China. 258 cases were recorded from the province of Hubei alone, 14 from the province of Guangdong, 05 from the municipality of Beijing and 01 from Shanghai municipality, among 278 confirmed in China [25]. Of the 278 confirmed cases, 51 are seriously ill, 12 are critically ill and 06 death from the city of Wuhan were reported. On 24th January, the total confirmed cases of 2019-nCoV cases expanded to 846 globally [26]. 830 cases of China and 375 cases of Hubei Province have been reported. 177 cases of serious illness and 25 deaths have been recorded from 830 cases. The detailed situation report of COVID-19 disease from 21 st January 2020 to 25th January 2021 has been discussed in section SARS-CoV2: A situation report. The chronology of 2019 novel Coronavirus events as monitored by the Wuhan Municipal Health Commission was illustrated in fig. 2 [27].

Additionally, fig. 3 is the World Health Organization (WHO) timeline for the 2019-nCoV since 31st December 2019 [28]. The WHO declares it a global public health emergency on 30th January 2020 and labeled it as a 2019 novel Coronavirus (2019-nCoV) [29]. 2019$\mathrm{nCoV}$ isolated from one patient and ultimately tested and expected as a causative agent in 16 other patients [30]. The International Committee of Taxonomy of Viruses (ICTV) has designated 2019nCoV as SARS-CoV-2 [31]. Based on these reports, snakes [32] or pangolins [33] may be the intermediary carriers but the true source of COVID-19 is still unknown according to the WHO (fig. 4) [33, 34]. On $11^{\text {th }}$ February 2020, the WHO officially named Coronavirus Disease-2019 (COVID-19) [36]. WHO later announced the COVID-19 outbreak as a pandemic on $11^{\text {th }}$ March 2020 [36, 37]. This infectious disease produces a pandemic climate that results in a growing number of cases of death, intense pressure on health care systems worldwide, and unparalleled movement, transit, industry, and educational constraints [39]. COVID-19 pandemic is a rapidly moving topic of research, which entraps the whole world gripping into a lockdown phase at the cost of millions of lives and restricting all means of travel synchronously. If COVID-19 spreads are not efficiently managed, the effects on global health services may pose considerable challenges and far-reaching consequences for the global economy [40].

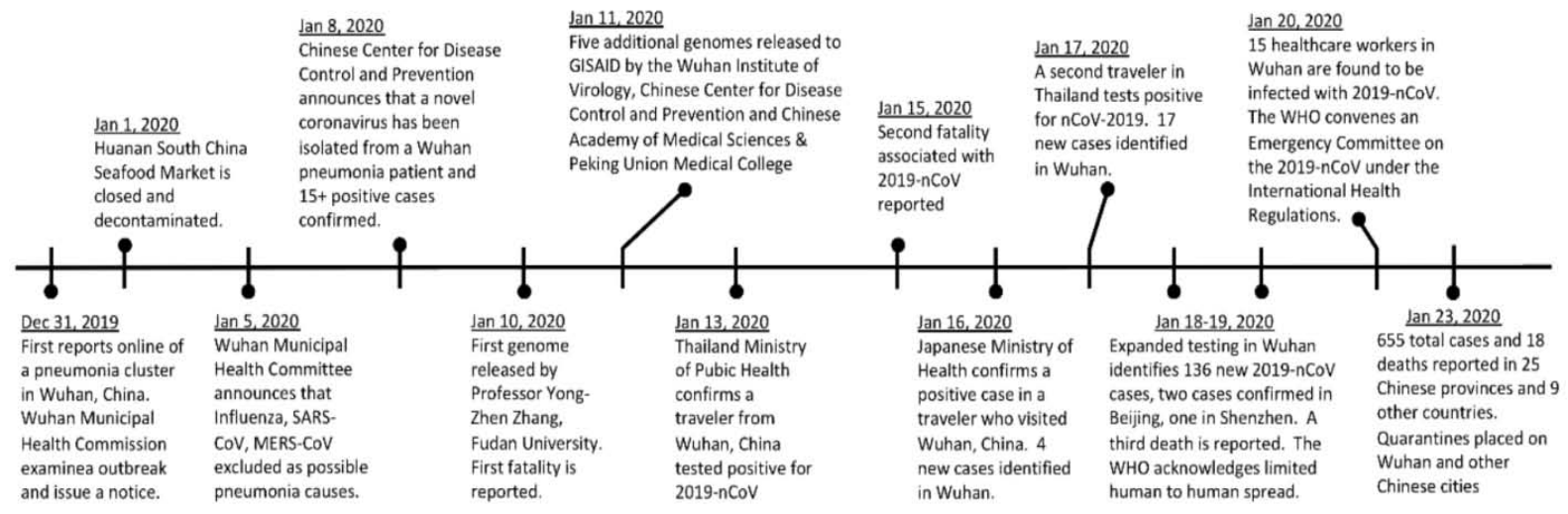

Fig. 2: 2019-nCoV events as monitored by the Wuhan Municipal Health Commission [27]

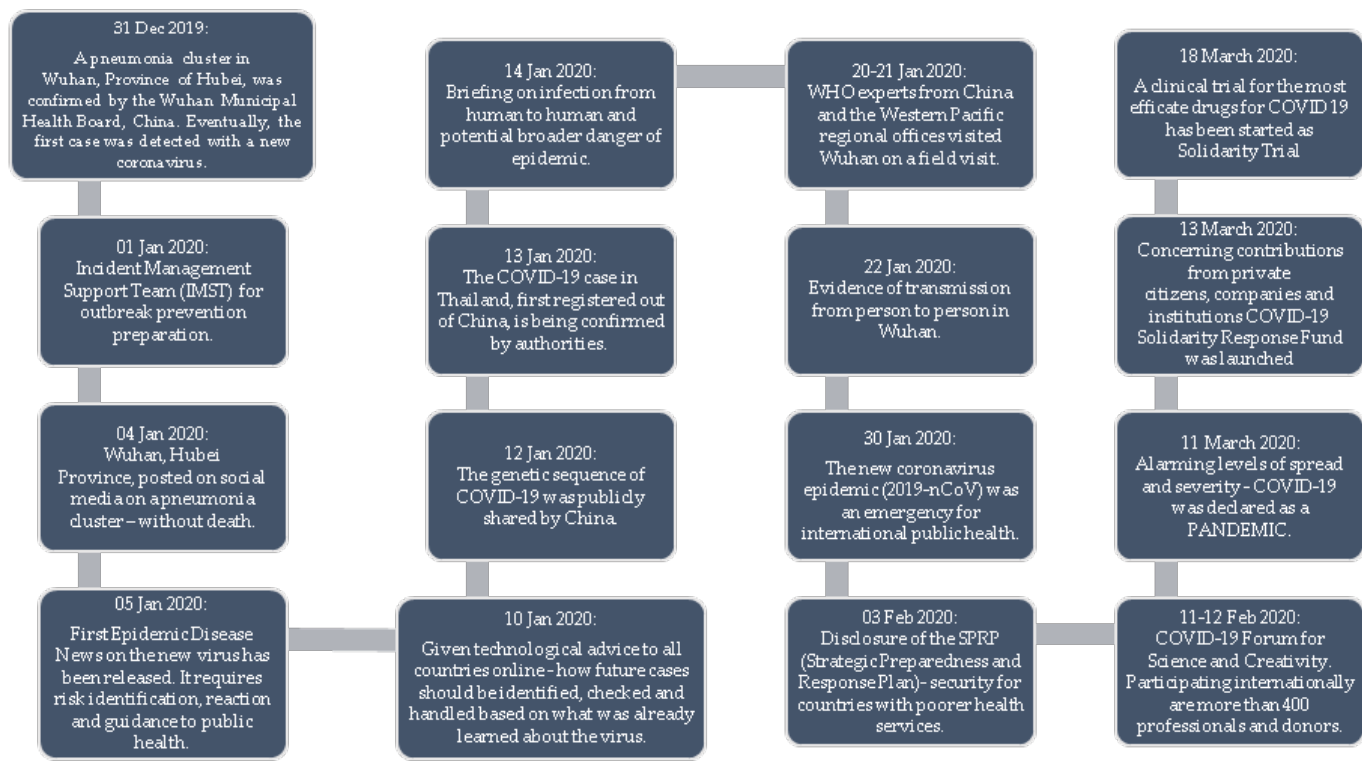

Fig. 3: WHO timeline for COVID-19 [28] 


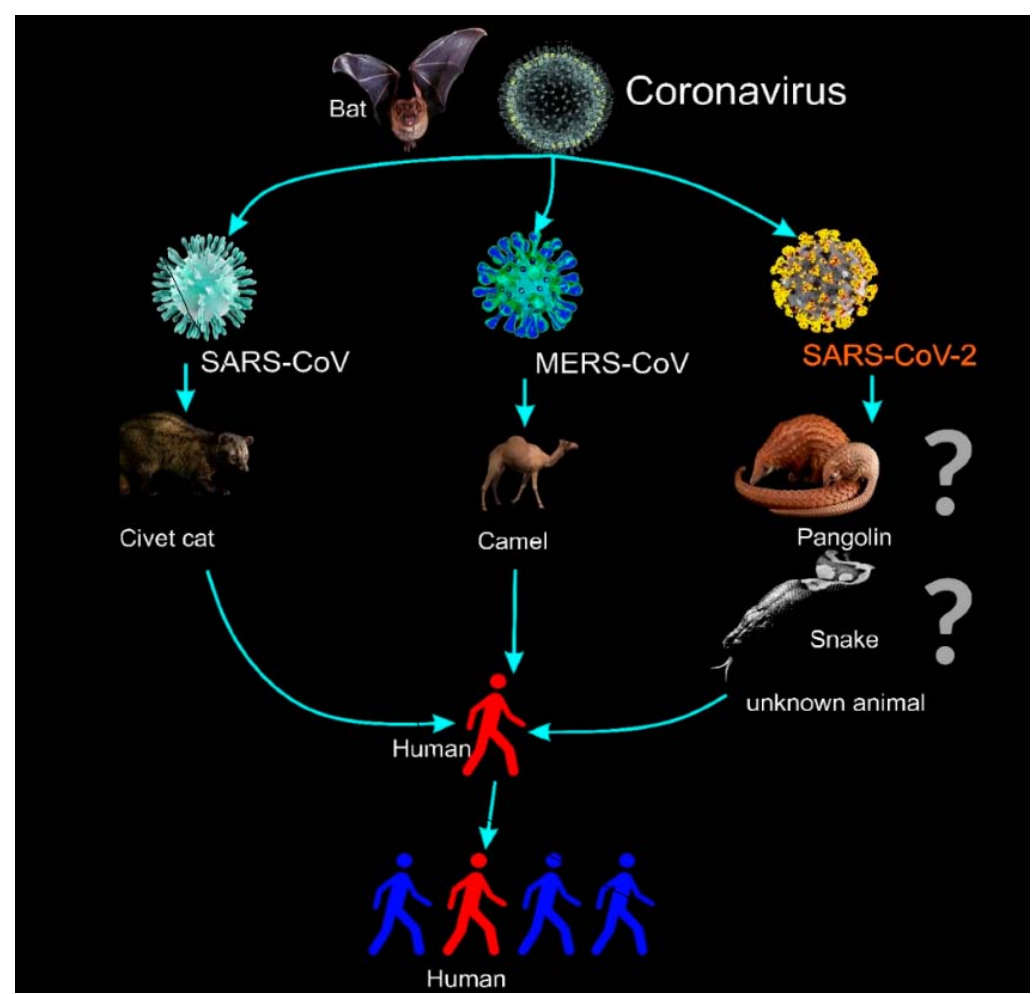

Fig. 4: Coronaviruses transmission from Animal to Human. However, SARS-CoV-2 or 2019-nCoV source is still unknown [35]

\section{Vulnerable impact on population}

The first 2019-nCoV sequence was made available online one day after the announcement was made on behalf of Dr. Yong-Zhen Zhang and scientists at the Fudan University in Shanghai [42]. The GSAID archive had five more 2019-nCoV sequences from institutes in China deposited on 11th January 2019 [26, 42]. With minimal patient records, robust declarations on communities that could be more vulnerable to $2019-\mathrm{nCoV}$ are sometimes difficult to produce. However, the severity of disease arising from SARS-CoV and MERS$\mathrm{CoV}$ was highly associated with the underlying circumstances of host age, sexuality, and overall health condition [44]. The new pathogen had $\sim 80 \%, \sim 50 \%$, and the genome of SARS-CoV, MERS-CoV, and bat $\mathrm{CoV}$ (RatG13), respectively, were found to have a similarity of $\sim 96 \%$ $[44,45]$. 2019-nCoV severe disease was associated with elderly patients ( $>60 \mathrm{y}$ old) that caused death [47]. The patient's underlying health conditions frequently play a vital role in its ultimate vulnerability. The 2019-nCoV had a severe vulnerable impact on patients suffering from health conditions such as hypertension, diabetes, and heart or kidney problems [48]. Similarly, the MERS$\mathrm{CoV}$ outbreak was responsible for most of the deaths to the patient suffering health illness such as smoking, hypertension, diabetes, cardiovascular, and other chronicle disease [49]. Taking into consideration signs that can differ in some patients, while others have a fever, cough, tiredness, and other symptoms. The signs may be identical to fever or cold patients [50]. The human-to-human transmission happens in droplets, touches, and fomites during earlier outbreaks of SARS-CoV and MERS-CoV and suggests a comparable 2019-nCoV transmittal mode (WHO Situation Report-4) [51]. Direct touch and droplet diffusion are the most probable means of transmission [52]. A new study of 2019-nCoV aerosols and surface persistence has shown that aerosols $(<5 \mu \mathrm{m})$ have viruses for at least $3 \mathrm{~h}$ and maybe more robust on plastic and stainless steel than on copper and carton board [52,53].

The United States' WHO and CDC [55] have reported that infectious persons with transmitting confirmations (fig. 4) are being spread from three-person cases outside China, mostly in the United States [47], Germany [56], and Vietnam [57]. The COVID-19 epidemic has expanded worldwide to 218 countries and territories (fig. 5) and has affected more than $101,441,979$ people worldwide, claiming more than 2,184,283 death by 28th January 2021 [58]. Fig. 5 represents COVID-19 cases per 01 million populations reported in the last $07 \mathrm{~d}$ globally from 28th December 2020 to 03rd January 2021[59]. China's death toll has surpassed the 2002-2003 SARS outbreaks and increased to 4,632 by $24^{\text {th }}$ April 2020 (WHO Situation Report-48) [60]. Therefore, SARS-CoV-2 is likewise more infectious and deadly than SARS-CoV [61]. At least 50 million people are locked up in China to slow down the propagation of COVID-19 [62]. Italy also took the same action on 8th March 2020 with 16 million residents in the northern part of the country under lockdown [63]. Since several mild and asymptomatic cases were not detected, the overall number of confirmed COVID-19 infections is underestimated [64]. Asymptomatic cases were estimated at 17.9 percent in the case report of the international transport cruise ship Diamond Princess in Japanese territorial waters (fig. 6) [65]. Asymptomatic patients are as contagious as symptomatic people and are hence able to further transmit the illness [66].

The U. S. Food and Drug Administration (FDA) has approved COVID-19 therapies and/or vaccines [67]. COVID-19 patients express signs that are unspecific and cannot be detected correctly. Guan et al. [68] recorded that $44 \%$ of 1,099 COVID-19 Chinese patients had a fever at the hospital and $89 \%$ developed a fever in the hospital. Patients had contaminants $(68 \%)$, exhaustion (38\%), development of sputum (34\%), and shortness of respiration (19\%). Many of these signs can be linked with other breathing infections. At present, diagnostic methods are important in the containment of COVID-19, which makes it possible to enforce restrictions restricting delivery, separation, and contact by case detection [50]. Currently, the main approach for testing and diagnosing COVID-19 disease is nucleic acid [69] and computed tomography (CT) testing [70]. As seen in fig. 7, the current workflow for diagnosis for COVID-19 [50]. 


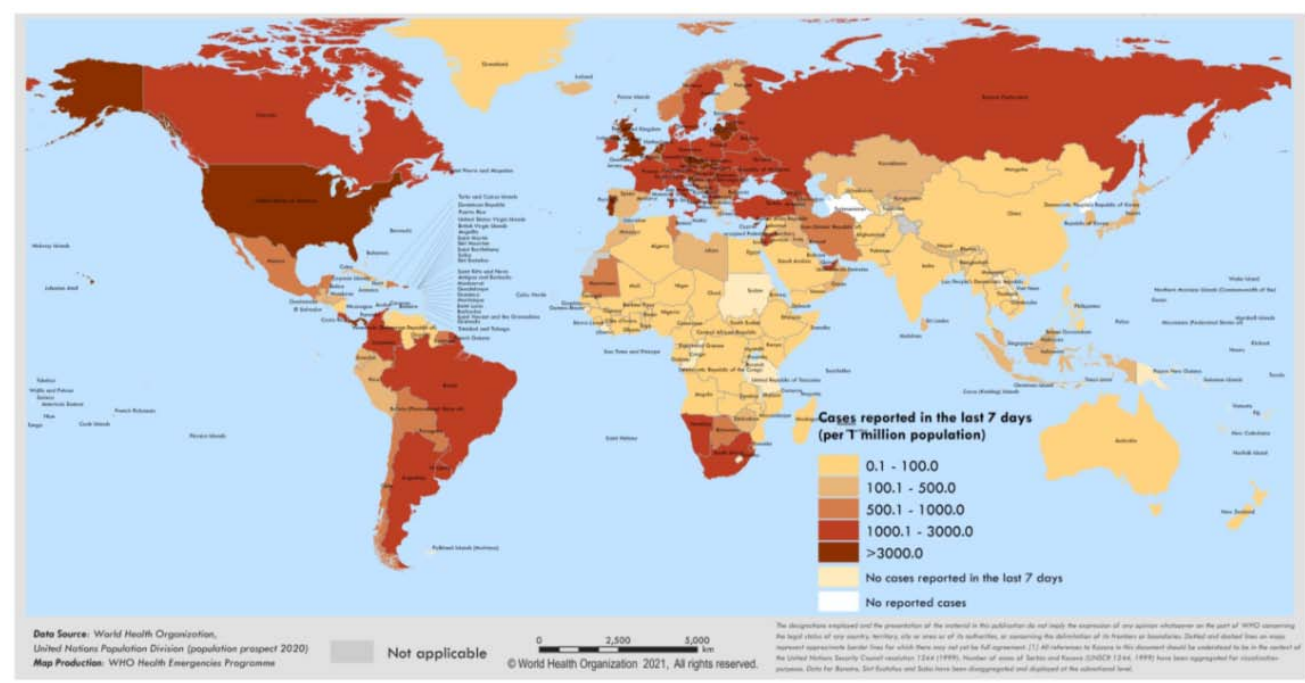

Fig. 5: World map showing the global distribution of COVID-19 confirmed cases per 01 million population from 28 December 2020 to 03 January 2021 [71]

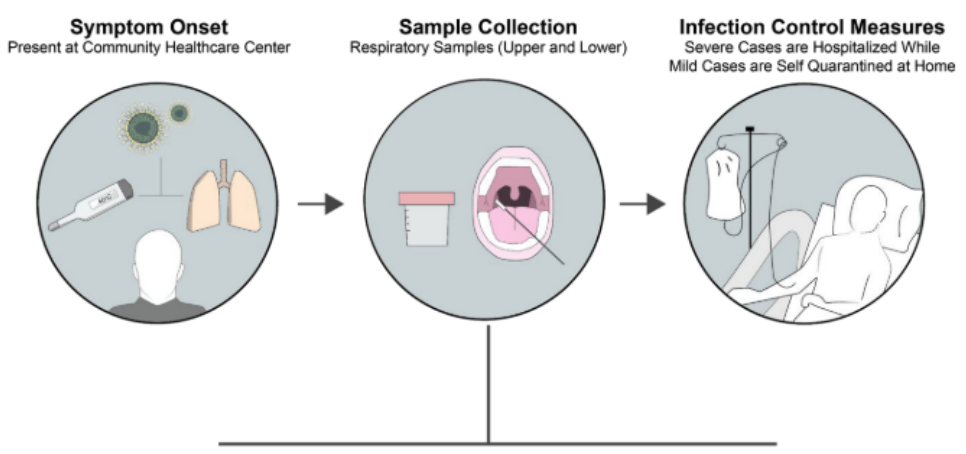

Sample Workflow

1. Collect Sample

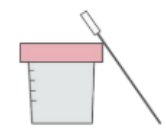

2. Transport Sample
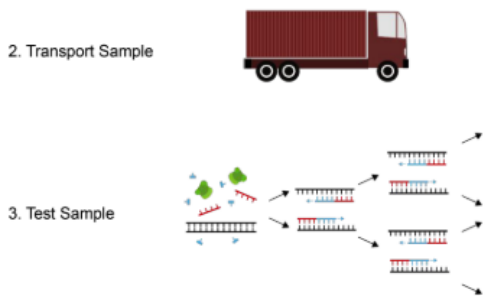

4. Sequence Sample
(Optional)

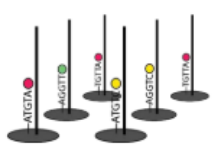

Fig. 6: COVID-19 diagnostic workflow [50]

\section{Contagiousness of the SARS-COV-2 or 2019-nCoV}

Three parameters that assess the extent of risk associated with this new coronavirus:

\section{a) Rate of transmission $\left(R_{0}\right)$-Proportion of newly infected} persons from a single case

The attack or transmission rate of a virus is suggested by its number (as quickly as the epidemic is spreading). The transmission rate $\left(R_{0}\right)$ measures the average number of individuals in whom the virus is transferred from some infected person [72]. An outbreak with a $R_{0}$ less than $1\left(\mathrm{R}_{0}<1\right)$ is gradually considered as disappeared. For the common flu, $\mathrm{R}_{0}$ was expected to be 1.3 and for SARS to be 2.0 [73]. Depending on preliminary studies the estimated value of $\mathrm{R}_{0}$ was between 1.5 and 3.5 [74-76]. On 23rd Jan. 2020, WHO's estimated the range of $\mathrm{R}_{0}$ in lying between 1.4 and 2.5 [77] Other studies have also estimated the range of $\mathrm{R}_{0}$ ranging from 3.6 to 4.0 and 2.24 to $3.58[78]$. 


\section{b) Case fatality rate (CFR)-The percentage of death cases}

CFR signifies the percentage of cases that actually die due to infection [79]. At the WHO press conference conducted on 29th January 2020, 2019-nCoV CFR was initially calculated at about $2 \%$ [80]. However, it was mentioned that it was too premature to be added a number to the mortality rate statistic without realizing how many were contaminated [80]. Wang et al. [14] gave a 3 percent early estimate for the total CFR. Dr. Tedros Adhanom Ghebreyesus, WHO Director-General, released on 3rd March 2020 that, "Globally, about 3.4\% of reported COVID-19 cases have died. By comparison, seasonal flu generally kills far fewer than $1 \%$ of those infected" [81]. However, the influenza pandemic of 1918 is projected to be less than five percent lethal $(\mathrm{CFR}<5 \%)$ yet to have major consequences because of widespread dissemination, and complacency is unfounded. Surveillance was raised not only in China but also globally as the disease turns into a pandemic [82]. On 20th Feb 2020 , a total of 55,924 laboratory cases were confirmed. Out of which 2114 people were died raising the CFR rate to $3.8 \%$. For zone, the CFR is averaged $5.8 \%$ in Wuhan versus $0.7 \%$ in other Chinese regions, respectively. In the earlier stages of the epidemic in the general CFR of China (17.3 percent of cases with symptoms during 1-10 January) have been decreased to 0.7 percent in the course of the duration after 1 February for patients with symptom initiation [83]. As stated by the NHC, China in a press conference on 4th Feb. 2020 , the nationwide mortality rate was $2.1 \%$ of confirmed cases [84]. The mortality rate was calculated using the formula:

\section{Mortality or CFR Rate = cumulative current total deaths/current confirmed cases}

There were $4.9 \%$ and $3.1 \%$ of mortality in Wuhan and Hubei Province. NHC has further stated that, from death analysis, more than $80 \%$ of people with an age of $60 \mathrm{y}$ are elderly and more than $75 \%$ have medical disorders such as coronary heart diseases and tumor diseases and more than $80 \%$ are old, with over $75 \%$ underlying health issues [82]. Elderly individuals with basic diseases had a greater clinical risk, whether or not they were infected by a coronavirus. For so long as they were, suffering from pneumonia, and the rate of fatality was also very high, and the rate of death of pneumonia has been not high due to infection. "Everyone must be explained at this point," the office of the NHC concluded [84]. According to epidemiologists, the fatality rate can be altered with the mutation of this virus [82]. At the moment the fatality rate is tested by the proportion of dividing the number of documented deaths by the confirmed cases. However, the result is not the actual case fatality rate. Moreover, the case fatality rate cannot currently be precisely estimated [79]. SARS-CoV originated in Beijing, China (November 2002-July 2003) has spread to 29 countries and caused 774 deaths of 8,096 infected persons ( 9.6 percent death rate). Given the SARS infection in China, on 29th January 2020, when Chinese officials confirmed 5,974 cases, Wuhan 2019-nCoV surpassed SARS (table 1). One day later on 30th January 2020, only the 8,096 reports that became the latest SARS outbreaks of 2003 became outpaced by fresh coronavirus infections worldwide. Of the 2,494 persons infected, MERS-CoV (in 2012) killed 858 people (34.4 percent fatality rates) [73].

Table 1: CFR of 2019-nCoV in comparison to others

\begin{tabular}{lll}
\hline Virus & CFR & References \\
\hline $2019-\mathrm{nCoV}$ & $3.4 \%$ (estimated) & {$[79]$} \\
SARS & $9.6 \%$ & {$[82]$} \\
MERS & $34 \%$ & {$[82]$} \\
Swine Flu & $0.02 \%$ & {$[82]$} \\
\hline
\end{tabular}

\section{c) COVID-19 incubation period (IbP)}

It is calculated that the IbP (a period from exposure to symptoms development) for the virus ranges from 2 to $14 \mathrm{~d}$ based on the following sources:

- The WHO recorded a time of incubation of COVID-19 between 2 and $10 \mathrm{~d}$ (WHO Situation Report-7)[85]
- Initially, the Chinese National Health Commission predicted an incubation period of 10 to $14 \mathrm{~d}$ [84].

- Incubation periods for COVID-19 disease are estimated between 2 and $14 \mathrm{~d}$ by the United States Centres for the Control and Prevention of Diseases (CDC). People with symptoms such as cough, breathing difficulties, fever, chills, muscle aches, sore throat, loss of taste or scent may suffer COVID-19 [86].

- DXY. cn, China's leading online medical and healthcare group recorded a "3-7 d up to $14 \mathrm{~d}$ " incubation duration [87].

The virus is spreading during the incubation period, but the patient presents no signs (asymptomatic transmission). It is very critical that health officials recognize the incubation time, monitor and potentially deter the transmission of the virus, and establish a more robust Quaranto scheme for individuals suspected of having the virus [88]. Backer et. al. results were reviewed on 88 cases of documented travel background (to and from) Wuhan, identified as contaminated by COVID-19 from 20th to 28th January. The overall incubation time was expected to be $6.4 \mathrm{~d}$. The IbP varies from 2.1 to $11.1 \mathrm{~d}$. The 11.1-day limit should, however, be deemed conservative [89]. The period of incubation varies considerably between patients. Hubei Province Local Government reported on 22nd February 2020 a case with an IbP of $27 \mathrm{~d}$ [90]. Furthermore, in a report documented by JAMA released on 21st February 2020, five cases with an incubation time of $19 \mathrm{~d}$ were reported [91]. Moreover, in a study on 09th February 2020, a 24-day incubation cycle was firstly observed [49]. WHO has said that this could represent a second exposure and not a longer duration of incubation. The average incubation time observed as showed in table 2 is focused on various case studies [88]. The incubation time for common influenza (seasonal flu) typically is about $2 \mathrm{~d}$ relative to other viruses. table 3 indicates the period of incubation for other coronaviruses [88].

Table 2: Mean incubation period

\begin{tabular}{lll}
\hline $\begin{array}{l}\text { Mean incubation } \\
\text { period (days) }\end{array}$ & Time duration (days) & References \\
\hline 3.0 & $0-24$ (on the basis 1324 cases) & {$[88]$} \\
5.2 & $4.1-7.0$ (on the basis 425 cases) & {$[88]$} \\
6.4 & $\begin{array}{l}2.1 \text { to 11.1 (based on travelers } \\
\text { from Wuhan) }\end{array}$ & {$[88]$} \\
\hline
\end{tabular}

Table 3: Incubation period of 2019-nCoVin comparison to others

\begin{tabular}{lll}
\hline Virus & IbP & References \\
\hline 2019-nCoV & $2-24$ d or 0-24 d & {$[88]$} \\
SARS & $2-7$ d (as long as $10 \mathrm{~d})$ & {$[88]$} \\
MERS & $5 \mathrm{~d}$ (as long as 2-14 d) & {$[88]$} \\
Swine Flu & $1-4 \mathrm{~d}$ (as long as 7 d) & {$[88]$} \\
Seasonal Flu & 2 d (as long as 1-4 d) & {$[88]$} \\
\hline
\end{tabular}

\section{SARS-CoV-2: A situation report}

Concerning WHO, a situation report on COVID-19 disease has been considered in this section from 21st January to 01st May 2020. On 04th April 2020, the worldwide confirmed cases climb above 1 million and death over 50,000 (WHO Situation Report-75) [92]. $13 \mathrm{~d}$ later, the sum of cases reported has universally crossed more than 2 million cases on 17th April 2020 (WHO Situation Report-88) [93]. The social and religious meetings of Ramadan are specifically influenced by physical distancing steps, such as shutting mosques, tracking public activities, and other constraints on the move. Therefore, on $15^{\text {th }}$ April 2020, WHO released a guideline on "Safe Practices in Ramadan" in the context of COVID-19 [94]. Its fundamental aim is to illustrate the guidance on public health in social and religious activities and meetings during Ramadan holy month that can be used in various national contexts [94]. Later on within $12 \mathrm{~d}$, the total number of reported cases has globally surpassed the 3 million mark on 29th April 2020 (WHO Situation Report-100) [95]. For 10 d interval, the total number of confirmed 
cases has globally exceeded 4 million marks as reported by Worldometer on 09th May 2020 [96]. Presently, the worldwide infection hits more than 100 million as stated by the Worldometer report on 25th January 2021 [97]. For the first 1 million cases to be recorded it took three months but has taken just four days for the global tally to grow that much again. This describes the severity of the pandemic community spread of COVID-19 disease and the necessity of required awareness to reduce its spread rate. Till 28th January 2021, the outbreak of COVID-19 disease has so far affected with 218 countries and territories across the globe and 02 international conveyances [98]. According to WHO, the number of cases globally is around triple that of severe influenza illness recorded annually. The details of the cases are given in table 4 . As reported by Worldometer on 28 ${ }^{\text {th }}$ January 2021 at 05:57 GMT, COVID-19 disease has globally infected 101,441,979 people, claimed over 2,184,283 lives and recovered 73,325,790 cases (table 4) [58]. Fig. 7 illustrates the list of the top 70 countries reported cases (reported/active) and death due to COVID-19 disease as of 25th January 2021. Table 5 provides a weekly epidemiological update on COVID-19 laboratory-confirmed cases and deaths founded on evidence from the situation report from the WHO, from $28^{\text {th }}$ December to $03^{\text {rd }}$ January 2021 [59].

Table 4: COVID-19 disease case details and condition status on 28th January 2021 at 10:22 GMT

\begin{tabular}{lllll}
\hline $\begin{array}{l}\text { Total confirmed cases } \\
\text { (Globally) }\end{array}$ & $\begin{array}{l}\text { Cases } \\
\text { type }\end{array}$ & Case status & Condition status & References \\
\hline $101,441,979$ & $\begin{array}{l}\text { Active } \\
\text { Cases }\end{array}$ & $\begin{array}{l}25,928,938 \\
\text { (Currently infected } \\
\text { patients) }\end{array}$ & $\begin{array}{l}25,821,571(99.6 \%) \\
110,335(0.4 \%)\end{array}$ & Mild Condition \\
& $\begin{array}{l}\text { Closed } \\
\text { Cases }\end{array}$ & $\begin{array}{l}75,513,041 \\
\text { (Cases which had }\end{array}$ & $73,325,790(97 \%)$ & Serious or critical \\
& & an outcome) & $2,184,283(3 \%)$ & Recovered/Discharged \\
& & & Deaths \\
\hline
\end{tabular}

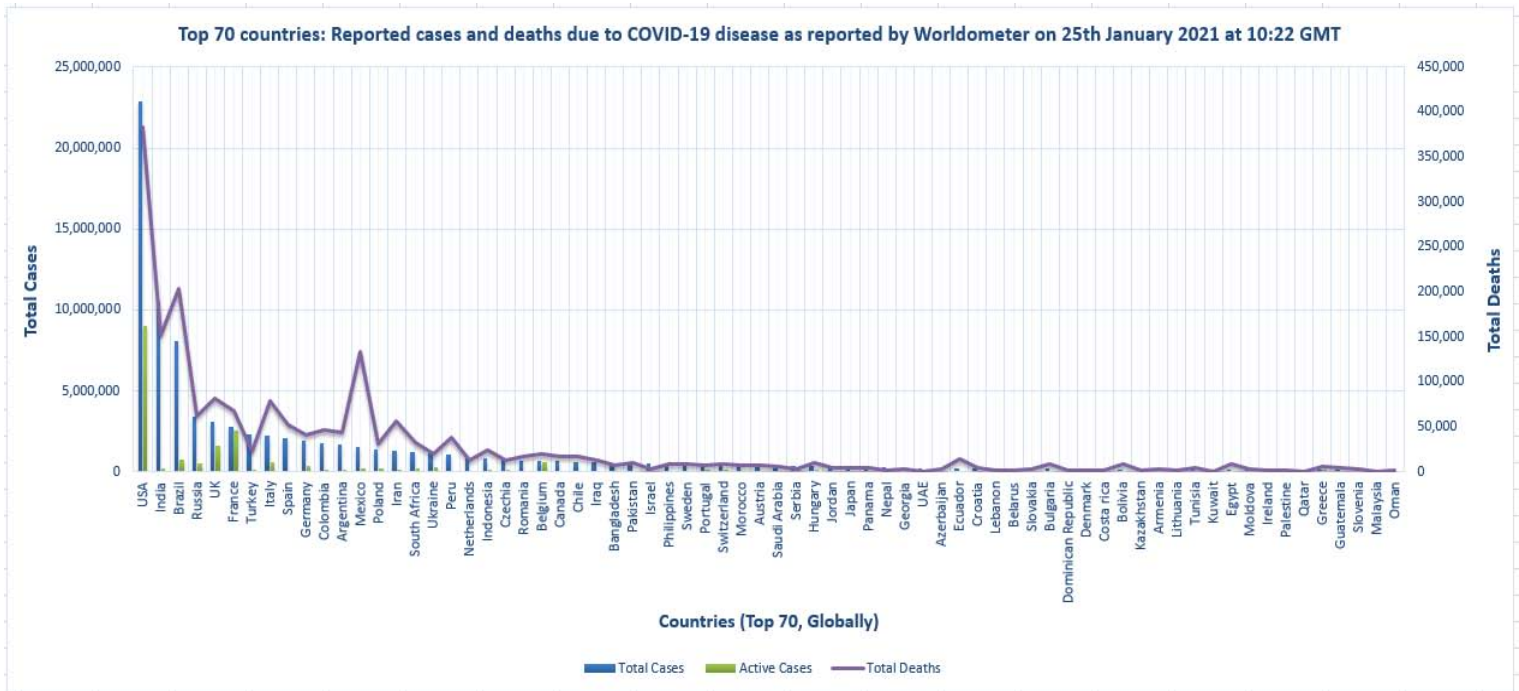

Fig. 7: Globally reported cases and deaths in top 70 countries, due to CoVID-19 disease as reported by Worldometer on 25 th January 2021

Table 5: Laboratory-confirmed COVID-19 case and death, epidemiological report 28th December to 3rd January 2021

\begin{tabular}{|c|c|c|c|c|c|c|}
\hline Location & New cases & $\begin{array}{l}\text { Cumulative } \\
\text { cases }\end{array}$ & New deaths & $\begin{array}{l}\text { Cumulative } \\
\text { deaths }\end{array}$ & $\begin{array}{l}\text { Type of } \\
\text { transmission }\end{array}$ & References \\
\hline Africa & 130007 & 1961234 & 3293 & 43592 & & [59] \\
\hline South Africa & 93978 & 1088889 & 2654 & 29175 & $\mathrm{COM}$ & [59] \\
\hline Nigeria & 5587 & 89163 & 55 & 1302 & $\mathrm{COM}$ & [59] \\
\hline Mauritania & 3393 & 14364 & 122 & 347 & $\mathrm{COM}$ & [59] \\
\hline Namibia & 3256 & 24654 & 26 & 213 & $\mathrm{COM}$ & [59] \\
\hline Ethiopia & 2636 & 125049 & 43 & 1944 & $\mathrm{COM}$ & [59] \\
\hline Algeria & 2302 & 100159 & 47 & 2769 & $\mathrm{COM}$ & [59] \\
\hline Uganda & 2149 & 35712 & 29 & 274 & $\mathrm{COM}$ & [59] \\
\hline Democratic Republic of the Congo & 1568 & 17848 & 25 & 591 & $\mathrm{COM}$ & [59] \\
\hline Zambia & 1559 & 21230 & 10 & 392 & COM & {$[59]$} \\
\hline Zimbabwe & 1528 & 14491 & 36 & 377 & $\mathrm{COM}$ & [59] \\
\hline Eswatini & 1344 & 9711 & 64 & 227 & COM & [59] \\
\hline Botswana & 1183 & 14805 & 4 & 42 & $\mathrm{COM}$ & [59] \\
\hline Senegal & 988 & 19511 & 29 & 416 & $\mathrm{COM}$ & [59] \\
\hline Kenya & 835 & 96678 & 30 & 1685 & COM & [59] \\
\hline Mozambique & 806 & 18968 & 9 & 168 & COM & [59] \\
\hline Ghana & 778 & 55064 & 2 & 335 & COM & [59] \\
\hline Rwanda & 750 & 8567 & 26 & 98 & CLU & [59] \\
\hline Burkina Faso & 685 & 6940 & 9 & 86 & COM & [59] \\
\hline Mali & 652 & 7226 & 27 & 276 & $\mathrm{COM}$ & [59] \\
\hline
\end{tabular}




\begin{tabular}{|c|c|c|c|c|c|c|}
\hline Cameroon & 571 & 26848 & 0 & 448 & COM & [59] \\
\hline Angola & 459 & 17608 & 8 & 407 & COM & [59] \\
\hline Niger & 405 & 3208 & 13 & 102 & COM & [59] \\
\hline Malawi & 368 & 6711 & 4 & 192 & COM & [59] \\
\hline Eritrea & 328 & 1320 & 2 & 3 & SPR & [59] \\
\hline South Sudan & 250 & 3558 & 1 & 63 & COM & [59] \\
\hline Cabo Verde & 185 & 11883 & 1 & 113 & COM & [59] \\
\hline Chad & 183 & 2169 & 1 & 104 & COM & [59] \\
\hline Comoros & 149 & 864 & 6 & 13 & COM & [59] \\
\hline Guinea & 138 & 13784 & 1 & 81 & $\mathrm{COM}$ & [59] \\
\hline Madagascar & 134 & 17767 & 2 & 262 & $\mathrm{COM}$ & [59] \\
\hline Togo & 128 & 3683 & 0 & 68 & $\mathrm{COM}$ & [59] \\
\hline Gabon & 74 & 9571 & 0 & 64 & $\mathrm{COM}$ & [59] \\
\hline Seychelles & 73 & 284 & 0 & 0 & SPR & [59] \\
\hline Benin & 46 & 3251 & 0 & 44 & COM & [59] \\
\hline Equatorial Guinea & 41 & 5277 & 1 & 86 & COM & [59] \\
\hline Burundi & 29 & 833 & 0 & 2 & $\mathrm{COM}$ & [59] \\
\hline Central African Republic & 15 & 4963 & 0 & 63 & $\mathrm{COM}$ & [59] \\
\hline Sierra Leone & 11 & 2560 & 0 & 76 & COM & [59] \\
\hline Gambia & 10 & 3802 & 1 & 124 & $\mathrm{COM}$ & [59] \\
\hline Sao Tome and Principe & 10 & 1024 & 0 & 17 & $\mathrm{COM}$ & [59] \\
\hline Mauritius & 3 & 527 & 0 & 10 & CLU & [59] \\
\hline Congo & 0 & 6200 & 0 & 100 & $\mathrm{COM}$ & [59] \\
\hline Guinea-Bissau & 0 & 2447 & 0 & 45 & $\mathrm{COM}$ & [59] \\
\hline Lesotho & 0 & 2577 & 0 & 50 & COM & [59] \\
\hline Liberia & 0 & 1800 & 0 & 83 & COM & [59] \\
\hline United Republic of Tanzania & 0 & 509 & 0 & 21 & COM & [59] \\
\hline \multicolumn{7}{|l|}{ Territories } \\
\hline Reunion & 128 & 9037 & 0 & 42 & CLU & [59] \\
\hline Mayotte & 123 & 5890 & 1 & 55 & CLU & [59] \\
\hline America & 1935621 & 36337439 & 32283 & 872486 & & \\
\hline USA & 1325424 & 19974413 & 17239 & 345253 & COM & [59] \\
\hline Brazil & 252018 & 7700578 & 4923 & 195411 & $\mathrm{COM}$ & [59] \\
\hline Colombia & 80173 & 1654880 & 1805 & 43495 & COM & [59] \\
\hline Mexico & 64942 & 1437185 & 4670 & 126507 & $\mathrm{COM}$ & [59] \\
\hline Argentina & 55040 & 1629594 & 897 & 43319 & COM & [59] \\
\hline Canada & 50966 & 587639 & 959 & 15679 & COM & [59] \\
\hline Panama & 23073 & 249733 & 308 & 4064 & COM & [59] \\
\hline Chile & 17508 & 615902 & 320 & 16724 & COM & [59] \\
\hline Peru & 11653 & 1017199 & 356 & 37724 & $\mathrm{COM}$ & [59] \\
\hline Bolivia & 8465 & 162055 & 103 & 9186 & COM & [59] \\
\hline Dominican Republic & 7025 & 172965 & 12 & 2416 & COM & [59] \\
\hline Costa Rica & 5259 & 169321 & 82 & 2185 & COM & [59] \\
\hline Ecuador & 5239 & 214513 & 63 & 14059 & COM & [59] \\
\hline Paraguay & 4461 & 108349 & 108 & 2262 & COM & [59] \\
\hline Honduras & 4315 & 122974 & 99 & 3160 & COM & [59] \\
\hline Uruguay & 3905 & 19753 & 50 & 193 & $\mathrm{COM}$ & [59] \\
\hline Guatemala & 3145 & 138316 & 64 & 4827 & COM & [59] \\
\hline Venezuela & 1959 & 113562 & 18 & 1028 & COM & [59] \\
\hline El Salvador & 1623 & 46242 & 54 & 1351 & COM & [59] \\
\hline Cuba & 1187 & 12225 & 5 & 146 & CLU & [59] \\
\hline Suriname & 391 & 6277 & 4 & 123 & CLU & [59] \\
\hline Belize & 317 & 10807 & 21 & 249 & $\mathrm{COM}$ & [59] \\
\hline Jamaica & 247 & 12931 & 9 & 303 & COM & [59] \\
\hline Haiti & 231 & 10077 & 1 & 236 & $\mathrm{COM}$ & [59] \\
\hline Bahamas & 83 & 7871 & 1 & 170 & CLU & [59] \\
\hline Guyana & 62 & 6351 & 2 & 164 & CLU & [59] \\
\hline Trinidad and Tobago & 61 & 7158 & 2 & 127 & COM & [59] \\
\hline Barbados & 48 & 395 & 0 & 7 & CLU & [59] \\
\hline Saint Lucia & 48 & 353 & 0 & 5 & SPR & [59] \\
\hline Nicaragua & 39 & 4829 & 1 & 165 & $\mathrm{COM}$ & [59] \\
\hline Grenada & 18 & 134 & 0 & 0 & SPR & [59] \\
\hline Saint Vincent & 14 & 122 & 0 & 0 & SPR & [59] \\
\hline Antigua and Barbuda & 4 & 159 & 0 & 54 & SPR & [59] \\
\hline Saint Kitts and Nevis & 3 & 33 & 0 & 0 & SPR & [59] \\
\hline Dominica & 0 & 96 & 0 & 0 & CLU & [59] \\
\hline \multicolumn{7}{|l|}{ Territories } \\
\hline Puerto Rico & 5489 & 77932 & 94 & 1526 & COM & [59] \\
\hline French Guiana & 500 & 13273 & 0 & 71 & COM & [59] \\
\hline Aruba & 214 & 5442 & 2 & 49 & COM & [59] \\
\hline Curacao & 179 & 4230 & 2 & 14 & COM & [59] \\
\hline Sint Maarten & 64 & 1434 & 1 & 27 & COM & [59] \\
\hline Turks and Caicos Islands & 64 & 908 & 0 & 6 & CLU & [59] \\
\hline United States Virgin Islands & 57 & 2036 & 0 & 23 & COM & [59] \\
\hline
\end{tabular}




\begin{tabular}{|c|c|c|c|c|c|c|}
\hline Bermuda & 43 & 604 & 1 & 10 & CLU & [59] \\
\hline Cayman Islands & 20 & 338 & 0 & 2 & SPR & [59] \\
\hline Martinique & 19 & 6091 & 1 & 43 & COM & [59] \\
\hline Saint Martin & 9 & 995 & 0 & 12 & COM & [59] \\
\hline Bonaire, Saint Eustatius and Saba & 7 & 189 & 0 & 3 & NA & [59] \\
\hline Anguilla & 3 & 15 & 0 & 0 & SPR & [59] \\
\hline Saint Barthélemy British & 1 & 190 & 0 & 0 & SPR & [59] \\
\hline British Virgin Islands & 0 & 93 & 0 & 1 & CLU & [59] \\
\hline Falkland Islands & 0 & 29 & 0 & 0 & No Cases & [59] \\
\hline Guadeloupe & 0 & 8620 & 0 & 155 & $\mathrm{COM}$ & [59] \\
\hline Montserrat & 0 & 13 & 0 & 1 & No Cases & [59] \\
\hline Saint Pierre and Miquelon & 0 & 16 & 0 & 0 & SPR & [59] \\
\hline Eastern Mediterranean & 154695 & 4977852 & 3057 & 122061 & & \\
\hline Iran & 42511 & 1237474 & 864 & 55438 & COM & [59] \\
\hline Lebanon & 16936 & 186408 & 97 & 1476 & COM & [59] \\
\hline Pakistan & 14880 & 484362 & 442 & 025 & CLU & [59] \\
\hline Tunisia & 11749 & 141373 & 339 & 4765 & COM & [59] \\
\hline Morocco & 11579 & 442141 & 248 & 7452 & CLU & [59] \\
\hline United Arab Emirates & 10749 & 211641 & 19 & 674 & COM & [59] \\
\hline Jordan & 10312 & 296668 & 148 & 3877 & $\mathrm{COM}$ & [59] \\
\hline Egypt & 9563 & 140878 & 389 & 7741 & CLU & [59] \\
\hline Iraq & 6254 & 597033 & 62 & 12829 & $\mathrm{COM}$ & [59] \\
\hline Libya & 3091 & 100744 & 72 & 1487 & $\mathrm{COM}$ & [59] \\
\hline Bahrain & 1666 & 93184 & 1 & 352 & CLU & [59] \\
\hline Kuwait & 1625 & 151074 & 8 & 937 & COM & [59] \\
\hline Qatar & 1337 & 144240 & 1 & 245 & COM & [59] \\
\hline Saudi Arabia & 913 & 362979 & 63 & 6239 & SPR & [59] \\
\hline Afghanistan & 861 & 52079 & 63 & 2221 & CLU & [59] \\
\hline Syrian Arab Republic & 684 & 11616 & 54 & 723 & COM & [59] \\
\hline Oman & 577 & 128867 & 8 & 1499 & COM & [59] \\
\hline Djibouti & 36 & 5841 & 0 & 61 & CLU & [59] \\
\hline Somalia & 24 & 4714 & 3 & 130 & SPR & [59] \\
\hline Yemen & 9 & 2105 & 4 & 611 & SPR & [59] \\
\hline Sudan & 0 & 23316 & 0 & 1468 & NA & [59] \\
\hline \multicolumn{7}{|l|}{ Territories } \\
\hline Occupied Palestinian territory & 9339 & 157879 & 172 & 1578 & COM & [59] \\
\hline Europe & 1553332 & 26885471 & 32898 & 588770 & & \\
\hline The United Kingdom & 343784 & 2599793 & 4165 & 74570 & $\mathrm{COM}$ & [59] \\
\hline Russian Federation & 186539 & 3236787 & 3728 & 58506 & CLU & [59] \\
\hline Germany & 124808 & 1765666 & 4494 & 34272 & CLU & [59] \\
\hline Italy & 102442 & 2141201 & 3365 & 74985 & CLU & [59] \\
\hline Turkey & 98662 & 1417697 & 1671 & 21295 & $\mathrm{COM}$ & [59] \\
\hline France & 91595 & 2599127 & 2346 & 64543 & COM & [59] \\
\hline Czechia & 69882 & 740481 & 916 & 11960 & $\mathrm{COM}$ & [59] \\
\hline Poland & 60763 & 1318562 & 2001 & 29119 & COM & [59] \\
\hline Netherlands & 59975 & 813725 & 600 & 11565 & COM & [59] \\
\hline Ukraine & 48104 & 1074093 & 1080 & 18854 & $\mathrm{COM}$ & [59] \\
\hline Portugal & 30874 & 423870 & 489 & 7045 & CLU & [59] \\
\hline Israel & 28963 & 425582 & 132 & 3338 & COM & [59] \\
\hline Romania & 23635 & 637395 & 811 & 15919 & $\mathrm{COM}$ & [59] \\
\hline Sweden & 22117 & 437379 & 68 & 8727 & COM & [59] \\
\hline Slovakia & 19940 & 187463 & 544 & 2317 & CLU & [59] \\
\hline Switzerland & 18879 & 450075 & 419 & 7049 & $\mathrm{COM}$ & [59] \\
\hline Serbia & 18537 & 341904 & 305 & 3288 & COM & [59] \\
\hline Spain & 16852 & 1893502 & 168 & 50442 & COM & [59] \\
\hline Denmark & 16374 & 167541 & 192 & 1345 & COM & [59] \\
\hline Lithuania & 16039 & 146637 & 390 & 1644 & COM & [59] \\
\hline Austria & 14604 & 362963 & 462 & 6214 & COM & [59] \\
\hline Belarus & 13203 & 198125 & 66 & 1442 & COM & [59] \\
\hline Hungary & 11935 & 327995 & 837 & 9884 & $\mathrm{COM}$ & [59] \\
\hline Ireland & 11532 & 96926 & 52 & 2252 & COM & [59] \\
\hline Slovenia & 10894 & 125086 & 359 & 2889 & CLU & [59] \\
\hline Belgium & 10458 & 650009 & 436 & 19693 & COM & [59] \\
\hline Croatia & 8028 & 212958 & 401 & 4072 & COM & [59] \\
\hline Georgia & 7564 & 229169 & 226 & 2603 & COM & [59] \\
\hline Azerbaijan & 6270 & 219462 & 249 & 2703 & CLU & [59] \\
\hline Latvia & 6110 & 41929 & 153 & 668 & CLU & [59] \\
\hline Kazakhstan & 5661 & 203563 & 96 & 2845 & CLU & [59] \\
\hline Bulgaria & 5496 & 202880 & 521 & 7644 & CLU & [59] \\
\hline Republic of Moldova & 4698 & 145694 & 137 & 3020 & $\mathrm{COM}$ & [59] \\
\hline Greece & 4595 & 139709 & 368 & 4921 & COM & [59] \\
\hline Cyprus & 4054 & 23445 & 18 & 129 & CLU & [59] \\
\hline Estonia & 3739 & 29131 & 40 & 244 & CLU & [59] \\
\hline Norway & 3346 & 48278 & 15 & 436 & CLU & [59] \\
\hline
\end{tabular}




\begin{tabular}{|c|c|c|c|c|c|c|}
\hline Albania & 3236 & 58991 & 47 & 1190 & CLU & [59] \\
\hline Bosnia and Herzegovina & 3015 & 112345 & 177 & 4100 & COM & [59] \\
\hline Armenia & 2880 & 160027 & 87 & 2850 & $\mathrm{COM}$ & [59] \\
\hline Armenia & 2439 & 49339 & 23 & 690 & CLU & [59] \\
\hline North Macedonia & 2364 & 83789 & 95 & 2522 & $\mathrm{COM}$ & [59] \\
\hline Finland & 2023 & 36107 & 37 & 561 & $\mathrm{COM}$ & [59] \\
\hline Luxembourg & 1062 & 46838 & 33 & 503 & $\mathrm{COM}$ & [59] \\
\hline Kyrgyzstan & 932 & 81305 & 11 & 1359 & CLU & [59] \\
\hline Malta & 756 & 12997 & 14 & 220 & CLU & [59] \\
\hline Uzbekistan & 487 & 77238 & 1 & 614 & CLU & [59] \\
\hline Andorra & 360 & 8166 & 1 & 84 & $\mathrm{COM}$ & [59] \\
\hline Liechtenstein & 223 & 2221 & 7 & 33 & SPR & [59] \\
\hline San Marino & 199 & 2463 & 4 & 61 & $\mathrm{COM}$ & [59] \\
\hline Monaco & 101 & 901 & 1 & 4 & SPR & [59] \\
\hline Iceland & 71 & 5754 & 1 & 29 & $\mathrm{COM}$ & [59] \\
\hline Holy See & 0 & 26 & 0 & 0 & SPR & [59] \\
\hline Tajikistan & 0 & 13182 & 0 & 89 & PND & [59] \\
\hline \multicolumn{7}{|l|}{ Territories } \\
\hline Kosovo & 1326 & 51688 & 35 & 1330 & $\mathrm{COM}$ & [59] \\
\hline Gibraltar & 678 & 2212 & 1 & 7 & CLU & [59] \\
\hline Jersey & 177 & 2760 & 3 & 44 & $\mathrm{COM}$ & [59] \\
\hline Faroe Islands & 43 & 614 & 0 & 0 & SPR & [59] \\
\hline Isle of Man & 6 & 380 & 0 & 25 & No Cases & [59] \\
\hline Guernsey & 2 & 299 & 0 & 13 & $\mathrm{COM}$ & [59] \\
\hline Greenland & 0 & 27 & 0 & 0 & No Cases & [59] \\
\hline South-East Asia & 208592 & 12051014 & 3756 & 184493 & & \\
\hline India & 136115 & 10323965 & 1813 & 149435 & CLU & [59] \\
\hline Indonesia & 51636 & 758473 & 1561 & 22555 & COM & [59] \\
\hline Bangladesh & 7085 & 515184 & 171 & 7599 & $\mathrm{COM}$ & [59] \\
\hline Myanmar & 4336 & 125616 & 132 & 2711 & CLU & [59] \\
\hline Sri Lanka & 3991 & 44371 & 24 & 211 & CLU & [59] \\
\hline Nepal & 3738 & 261438 & 51 & 1870 & CLU & [59] \\
\hline Thailand & 1359 & 7379 & 4 & 64 & CLU & [59] \\
\hline Maldives & 216 & 13834 & 0 & 48 & CLU & [59] \\
\hline Bhutan & 113 & 710 & 0 & 0 & CLU & [59] \\
\hline Timore-Leste & 3 & 44 & 0 & 0 & SPR & [59] \\
\hline Western Pacific & 52979 & 1112724 & 730 & 20288 & & \\
\hline Japan & 23642 & 240954 & 335 & 3548 & CLU & [59] \\
\hline Malaysia & 13473 & 117373 & 32 & 483 & CLU & [59] \\
\hline Philippines & 7911 & 476916 & 186 & 9523 & $\mathrm{COM}$ & [59] \\
\hline Republic of Korea & 6378 & 63244 & 154 & 962 & CLU & [59] \\
\hline China & 570 & 96894 & 14 & 4791 & CLU & [59] \\
\hline Australia & 166 & 28462 & 1 & 909 & SPR & [59] \\
\hline Mongolia & 160 & 1242 & 0 & 0 & CLU & [59] \\
\hline Singapore & 143 & 58662 & 0 & 29 & SPR & [59] \\
\hline Vietnam & 42 & 1482 & 0 & 35 & CLU & [59] \\
\hline New Zealand & 37 & 1825 & 0 & 25 & CLU & [59] \\
\hline Cambodia & 17 & 381 & 0 & 0 & SPR & [59] \\
\hline Brunei Darussalam & 5 & 157 & 0 & 3 & No Cases & [59] \\
\hline Fiji & 3 & 49 & 0 & 2 & SPR & [59] \\
\hline Lao People's Democratic Republic & 0 & 41 & 0 & 0 & SPR & [59] \\
\hline Papua New Guinea & 0 & 780 & 0 & 9 & COM & [59] \\
\hline Solomon Islands & 0 & 17 & 0 & 0 & No Cases & [59] \\
\hline \multicolumn{7}{|l|}{ Territories } \\
\hline French Polynesia & 376 & 16926 & 6 & 114 & SPR & [59] \\
\hline Guam & 54 & 7148 & 2 & 123 & CLU & [59] \\
\hline New Caledonia & 2 & 40 & 0 & 0 & SPR & [59] \\
\hline Marshall Islands & 0 & 4 & 0 & 0 & No Cases & [59] \\
\hline Northern Mariana Islands & 0 & 122 & 0 & 2 & PND & [59] \\
\hline Vanuatu & 0 & 1 & 0 & 0 & No Cases & [59] \\
\hline Wallis and Futuna & 0 & 4 & 0 & 0 & SPR & [59] \\
\hline Grand Total & 4035226 & 83326479 & 76017 & 1831703 & $\ldots$ & \\
\hline
\end{tabular}

Note:

Abbreviated: COM: Community; CLU: Clusters; SPR: Sporadic; PND: Pending; N. A: Not Applicable

The different degrees of transmission classification [99] are categorized as follows:

- Cases Sporadic in Nature: One or two instances either imported or observed locally.
- Cases Clusters in Nature: Cases of experience, grouped over time, geographical location, and/or general exposure.

- Community transmission in Nature: The history of broader local transmission outbreaks decided by empirical causes, including a large number of cases that cannot be correlated with transmission chains, a large number of cases resulting from the surveillance of labs, and/or numerous unrelated clusters across different areas of the country/territory/area. 
- Cases awaiting or pending in Nature: Transmission designation not referred by the WHO.

\section{SARS-CoV-2 in India: A state-wise analysis}

India is a large nation with $3,287,240$ square kilometers, a geographical area of nearly 1.3 billion inhabitants. The majority of Indian States are very large in geographic and urban areas so the transmission of the disease must be treated separately in each state where the conditions are quite different (table 8) [100]. A student returning from Wuhan on 30th January 2020 detected the first case of COVID 19. In different countries in the world, the number of cases has since steadily increased. More than 10 million infections are confirmed and are now propagated in India from 22nd January 2021 [101]. The first infection date and history of travel in each Indian state for the infected person presented in fig. 8. Indeed, for the first time, a traveler from one or more contaminated COVID-19 countries has been observed in every union and territory except Assam, Tripura, Nagaland, Meghalaya, and Arunachal Pradesh [102]. All 22nd March 2020, on international flights to India by the Indian government imposed a total ban. Fig. 8 justifies government policy to suspend transcontinental flight in contrast [103]. Table 6 displays the Indian Government's effective preventive steps for preventing the growth rate of COVID-19 [100].

Table 6: Significant protective steps to monitor the dissemination of COVID-19 by the Indian Government [100]

\begin{tabular}{|c|c|c|}
\hline Dates & Preventive measures adopted by Indian government & References \\
\hline $25^{\text {th }}$ January to $13^{\text {th }}$ March 2020 & Health Screening at Airports and Border crossings. & [100] \\
\hline $26^{\text {th }}$ February to $20^{\text {th }}$ March 2020 & Quarantine policies are introduced: step by step for travelers from various countries & {$[100]$} \\
\hline $26^{\text {th }}$ February to $13^{\text {th }}$ March 2020 & Restrictions to Visa & [100] \\
\hline $05^{\text {th }}$ March 2020 & Limitation to Public Gathering (Museums and religious places) & {$[100]$} \\
\hline $11^{\text {th }}$ March 2020 & Border Checks & {$[100]$} \\
\hline $13^{\text {th }}$ March 2020 to $15^{\text {th }}$ March 2020 & Border closure & {$[100]$} \\
\hline $16^{\text {th }}$ March 2020 & Limitation to Public Gathering & {$[100]$} \\
\hline $18^{\text {th }}$ March 2020 & Travel Restrictions & {$[100]$} \\
\hline 20 th March 2020 & Coronavirus disease testing & [100] \\
\hline \multirow[t]{2}{*}{ 22 nd March 2020} & Flight Suspension & {$[100]$} \\
\hline & Passenger Train Services canceled till 31st March 2020 & {$[100]$} \\
\hline 24th March 2020 & Flight Suspension (Domestic Airlines) & {$[100]$} \\
\hline \multirow[t]{2}{*}{$25^{\text {th }}$ March 2020} & 21-day complete lockdown in the whole country & {$[100]$} \\
\hline & Passenger Train Services canceled till 14th April 2020 & {$[100]$} \\
\hline $25^{\text {th }}$ March 2020 & Increase of Quarantine and Isolation facilities & {$[100]$} \\
\hline $14^{\text {th }}$ April 2020 & Lockdown Extended nationwide until 03rd May 2020 & {$[100]$} \\
\hline $01^{\text {st }}$ May 2020 & Lockdown Extended nationwide until 17th May 2020 & {$[100]$} \\
\hline
\end{tabular}

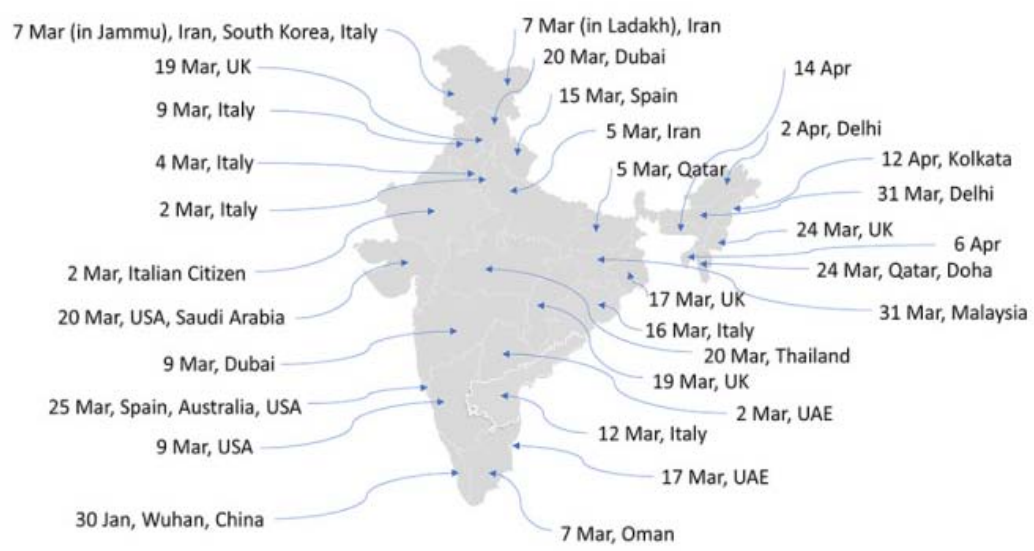

Fig. 8: The arrival of the first case in each State of India [100]

Day Wise Cases: India

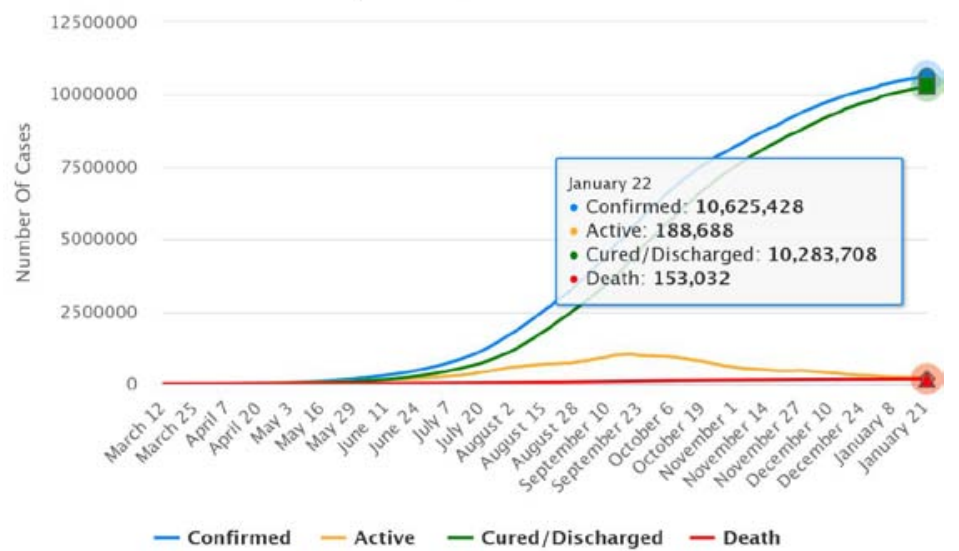

Fig. 9: Day wise different cases in India, 22nd January 2021 [101] 
Presently, as COVID-19 statistics on 22nd January 2021, Maharashtra, Karnataka, Andhra Pradesh, Kerala, Tamil Nadu, Delhi, Uttar Pradesh, West Bengal, Odisha, and Rajasthan are among the top 10 states holding the most number of confirmed cases. The growth rate seems to have dropped in Kerala, as reported by the first COVID-19 event [104]. Table 7 presents the state-wise status of
COVID-19 cases in India until 22nd January 2021[101]. Considering the top 10 most affected states in India among the 36 based on confirmed cases, active cases, cured cases, and death are presented in table 8. Fig. 9 illustrates the day-wise different types of cases in India and fig. 10 represents state-wise data of COVID-19 disease.

Table 7: Status of COVID-19 disease in India-state-wise data until 22 January 2021[101]

\begin{tabular}{|c|c|c|c|c|c|c|}
\hline S. No. & State & Confirmed cases & Active cases & Cured/discharged cases & Deaths & References \\
\hline 1 & Andaman and Nicobar Islands & 4991 & 25 & 4904 & 62 & {$[101]$} \\
\hline 2 & Andhra Pradesh & 886557 & 1522 & 877893 & 7142 & [101] \\
\hline 3 & Arunachal Pradesh & 16816 & 38 & 16722 & 56 & [101] \\
\hline 4 & Assam & 216940 & 2568 & 213295 & 1077 & {$[101]$} \\
\hline 5 & Bihar & 258414 & 2923 & 254023 & 1468 & {$[101]$} \\
\hline 6 & Chandigarh & 20639 & 146 & 20162 & 331 & [101] \\
\hline 7 & Chhattisgarh & 295509 & 5638 & 286277 & 3594 & [101] \\
\hline 8 & $\begin{array}{l}\text { Dadra and Nagar Haveli and } \\
\text { Daman and Diu }\end{array}$ & 3393 & 10 & 3381 & 2 & [101] \\
\hline 9 & Delhi & 633276 & 2120 & 620374 & 10782 & {$[101]$} \\
\hline 10 & Goa & 52712 & 865 & 51090 & 757 & {$[101]$} \\
\hline 11 & Gujarat & 257813 & 5491 & 247950 & 4372 & [101] \\
\hline 12 & Haryana & 266819 & 1679 & 262140 & 3000 & {$[101]$} \\
\hline 13 & Himachal Pradesh & 57121 & 556 & 55595 & 970 & {$[101]$} \\
\hline 14 & Jammu and Kashmir & 123764 & 1111 & 120729 & 1924 & {$[101]$} \\
\hline 15 & Jharkhand & 118079 & 1032 & 115989 & 1058 & [101] \\
\hline 16 & Karnataka & 934252 & 7573 & 914492 & 12187 & [101] \\
\hline 17 & Kerala & 870529 & 69998 & 796986 & 3545 & [101] \\
\hline 18 & Ladakh & 9673 & 73 & 9471 & 129 & [101] \\
\hline 19 & Lakshadweep & 48 & 48 & 0 & 0 & [101] \\
\hline 20 & Madhya Pradesh & 252767 & 4599 & 244392 & 3776 & {$[101]$} \\
\hline 21 & Maharashtra & 2000878 & 46836 & 1903408 & 50634 & [101] \\
\hline 22 & Manipur & 28938 & 220 & 28351 & 367 & [101] \\
\hline 23 & Meghalaya & 13721 & 129 & 13446 & 146 & [101] \\
\hline 24 & Mizoram & 4349 & 64 & 4276 & 9 & [101] \\
\hline 25 & Nagaland & 12070 & 107 & 11875 & 88 & [101] \\
\hline 26 & Odisha & 333866 & 1418 & 330545 & 1903 & {$[101]$} \\
\hline 27 & Puducherry & 38772 & 299 & 37830 & 643 & [101] \\
\hline 28 & Punjab & 171316 & 2343 & 163438 & 5535 & [101] \\
\hline 29 & Rajasthan & 316081 & 3934 & 309391 & 2756 & [101] \\
\hline 30 & Sikkim & 6062 & 148 & 5783 & 131 & [101] \\
\hline 31 & Tamil Nadu & 833011 & 5196 & 815516 & 12299 & [101] \\
\hline 32 & Telangana & 292835 & 3781 & 287468 & 1586 & [101] \\
\hline 33 & Tripura & 33342 & 40 & 32911 & 391 & [101] \\
\hline 34 & Uttar Pradesh & 597823 & 7717 & 581509 & 8597 & [101] \\
\hline 35 & Uttarakhand & 95354 & 1876 & 91852 & 1626 & [101] \\
\hline 36 & West Bengal & 566898 & 6565 & 550244 & 10089 & [101] \\
\hline India & & 10625428 & 188688 & 10283708 & 153032 & \\
\hline
\end{tabular}

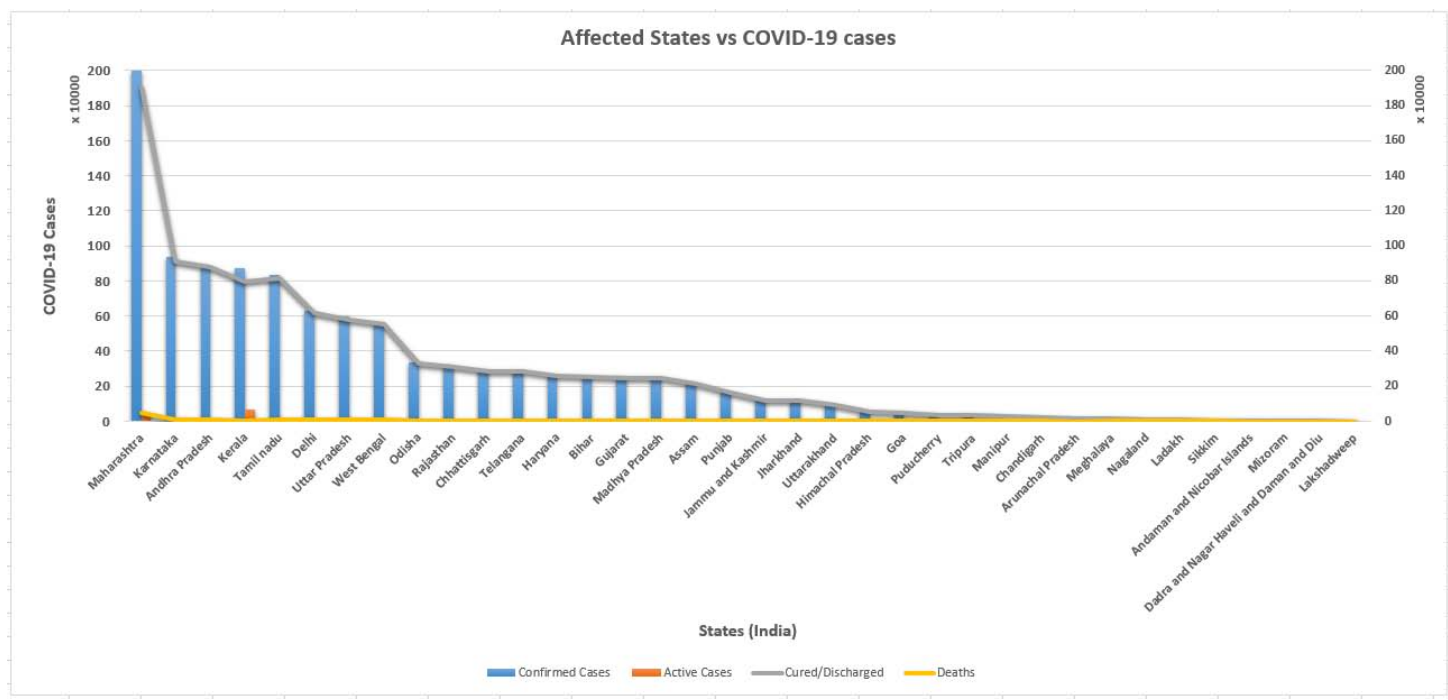

Fig. 10: Total cases of COVID-19 in various Indian states, 22 January 2021 
Table 8: Top 10 most affected states in India until 22 January 2021

\begin{tabular}{|c|c|c|c|c|}
\hline S. No. & Confirmed cases & Active cases & Cured cases & Deaths \\
\hline 1 & Maharashtra & Kerala & Maharashtra & Maharashtra \\
\hline 2 & Karnataka & Maharashtra & Karnataka & Tamil Nadu \\
\hline 3 & Andhra Pradesh & Uttar Pradesh & Andhra Pradesh & Karnataka \\
\hline 4 & Kerala & Karnataka & Tamil Nadu & Delhi \\
\hline 5 & Tamil Nadu & West Bengal & Kerala & West Bengal \\
\hline 6 & Delhi & Chhattisgarh & Delhi & Uttar Pradesh \\
\hline 7 & Uttar Pradesh & Gujarat & Uttar Pradesh & Andhra Pradesh \\
\hline 8 & West Bengal & Tamil Nadu & West Bengal & Punjab \\
\hline 9 & Odisha & Madhya Pradesh & Odisha & Gujarat \\
\hline 10 & Rajasthan & Rajasthan & Rajasthan & Madhya Pradesh \\
\hline
\end{tabular}

\section{Variants of SARS-CoV-2: A Recent Update}

The WHO Epidemiological report-COVID-19 published on $03^{\text {rd }}$ January 2021, stated for the different variants of SARS-CoV-2, the virus that causes COVID-19, has again raised interest in and concern about the impact of the viral changes. In the last months, two distinct variants of SARS-CoV-2 have been reported to WHO as unusual public health events from the United Kingdom of Great Britain and Northern Ireland, referred to as VOC 202012/01, and the Republic of South Africa named 501Y. V2[59]. Preliminary epidemiological, modeling, phylogenetic, and clinical conclusions indicate that SARS-CoV-2 VOC 202012/01 rises in transmissibility and preliminary results also indicate no improvements in the seriousness of the disease (as calculated by hospital duration and $28 \mathrm{~d}$ case fatality) or reinfection of variant cases in contrast to other circulating SARS-CoV-2 viruses in the United Kingdom[105]. To date, outside of the United Kingdom, 40 countries across five of the six WHO regions have reported cases of VOC 202012/01, while outside of South Africa six countries, in two of the six WHO regions have reported cases of 501Y. V2 [106]. Further epidemiological and virological investigations have been undertaken by the authorities in both countries to further determine the transmissibility of new variants such as seriousness, risk of reinfection, and antibody reaction, and their likely effect on countermeasures including diagnosis, treatment, and vaccine [107].

The decrease observed in the last week in new death has been reversed with deaths rising by $3 \%$ to 76,000 (fig. 11) [59]. The Region of the Americas accounted for $47 \%$ of all new cases and $42 \%$ of all new deaths globally in the past week. New cases and deaths remained high in the European Region, which accounted for 38\% and $43 \%$ respectively, showing a slight decrease in new cases and a slight increase in new death. Recent cases and deaths continue to decline in the South-East Asia and Eastern Mediterranean regions. In African Region, while both new cases and deaths remain low in absolute numbers, for the fourth week in a row, the Region is reporting the largest percentage increase globally in weekly reported case numbers and there was a further $13 \%$ increase in new cases and $28 \%$ increase in new deaths. At the beginning of the year 2021, COVID-19 vaccination campaigns have been initiated worldwide. The current epidemiological situation with near-record numbers of new cases and deaths makes it imperative to go on adhere to safety measures to prevent further transmission and loss of life. Five countries which reported the highest amount of cases were the USA (with 1,325,424 cases, just under a third of global cases), the UK of Great Britain and Northern Ireland (3,43,784 cases, with a rapid increase of $36 \%)$, Brazil $(2,52,018$ cases, an $11 \%$ decrease), the Russian Federation (1,86,539 cases, a 7\% decrease) and India $(1,36,115$ cases, a $13 \%$ decrease).

\section{Case distribution: Graphical Analysis}

\section{(a) Total confirmed cases (Globally)}

The graph describes the rise in 2019-ncoV confirmed cases globally from 21st January 2020 (282 cases) to 25th January 2021 $(100,283,922)$ [97] (fig. 12). The rise in confirmed cases begins after $16^{\text {th }}$ February 2020 and there has got a spike since 20th March 2020. Since then the total confirmed 2019-ncoV cases globally have been consistently increasing in a rapid form. The milestone for the worldwide-confirmed cases is given in table 9. Country-wise case distribution outside china and worldwide has been shown in fig. 13 and 14 (as reported by worldmeter, $17^{\text {th }}$ January 2021) $[95,106]$.

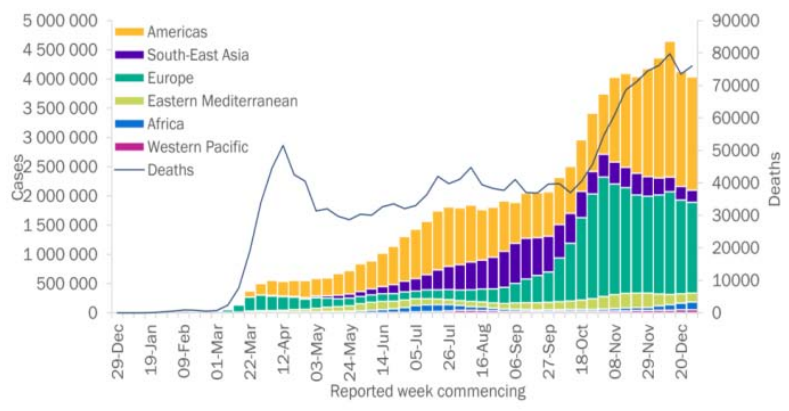

Fig. 11: Covid-19 global cases and deaths, reported weekly by who [59]

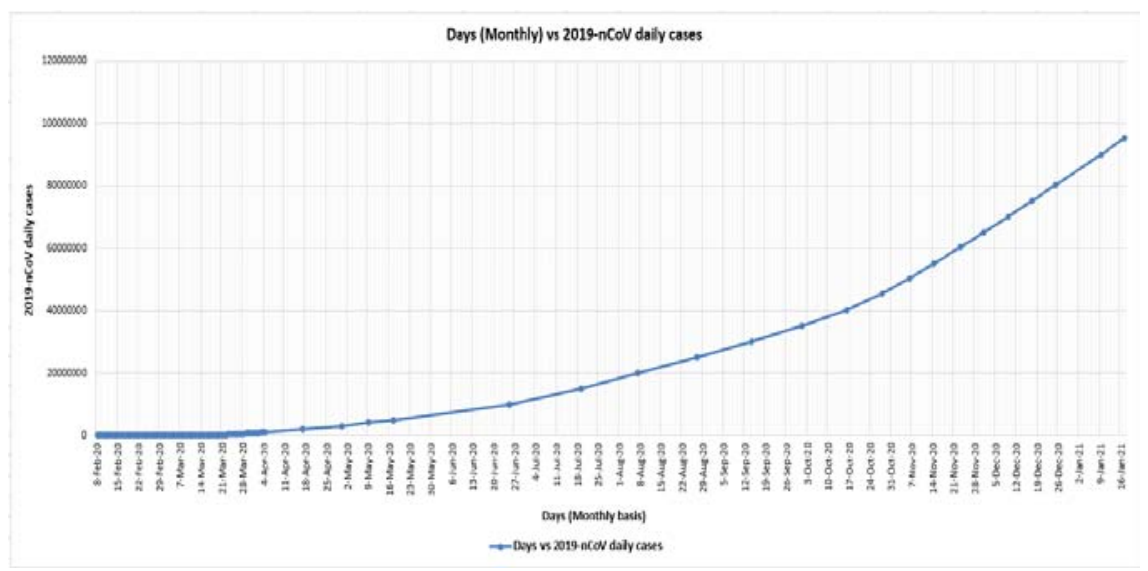

Fig. 12: 2019-nCoV confirmed cases globally, 17 ${ }^{\text {th }}$ January 2021 
Table 9: Milestone for 2019-nCoV Global Cases

\begin{tabular}{|c|c|}
\hline Dates & Milestone (Global Cases) \\
\hline 04th April 2020 & Exceeded 1 million (1051697 cases) \\
\hline 17 th April 2020 & Exceeded 2 million (2074529 cases) \\
\hline 29th April 2020 & Exceeded 3 million (3018681 cases) \\
\hline 09th May 2020 & Exceeded 4 million (4098288 cases) \\
\hline 17th May 2020 & Exceeded 5 million (5010291 cases) \\
\hline $25^{\text {th }}$ June 2020 & Exceeded 10 million ( 10024554 cases) \\
\hline 19th July 2020 & Exceeded 15 million ( 15051517 cases) \\
\hline 07th August 2020 & Exceeded 20 million (20014171 cases) \\
\hline $27^{\text {th }}$ August 2020 & Exceeded 25 million (25209843 cases) \\
\hline $14^{\text {th }}$ September 2020 & Exceeded 30 million ( 30143022 cases) \\
\hline $01^{\text {st }}$ October 2020 & Exceeded 35 million (35208481 cases) \\
\hline $16^{\text {th }}$ October 2020 & Exceeded 40 million ( 40270868 cases) \\
\hline 28 $8^{\text {th }}$ October 2020 & Exceeded 45 million ( 45510562 cases) \\
\hline 06 $6^{\text {th }}$ November 2020 & Exceeded 50 million (50421101 cases) \\
\hline 14th November 2020 & Exceeded 55 million (55170426 cases) \\
\hline $23^{\text {rd }}$ November 2020 & Exceeded 60 million ( 60412853 cases) \\
\hline 01 $1^{\text {st }}$ December 2020 & Exceeded 65 million ( 65070502 cases) \\
\hline 09th December 2020 & Exceeded 70 million (70107969 cases) \\
\hline 17th December 2020 & Exceeded 75 million ( 75330456 cases) \\
\hline $25^{\text {th }}$ December 2020 & Exceeded 80 million (80339800 cases) \\
\hline 02 $2^{\text {nd }}$ January 2021 & Exceeded 85 million (85075159 cases) \\
\hline $09^{\text {th }}$ January 2021 & Exceeded 90 million (90102438 cases) \\
\hline $17^{\text {th }}$ January 2021 & Exceeded 95 million (95520875 cases) \\
\hline $25^{\text {th }}$ January 2021 & Exceeded 100 million ( 100283922 cases) \\
\hline
\end{tabular}

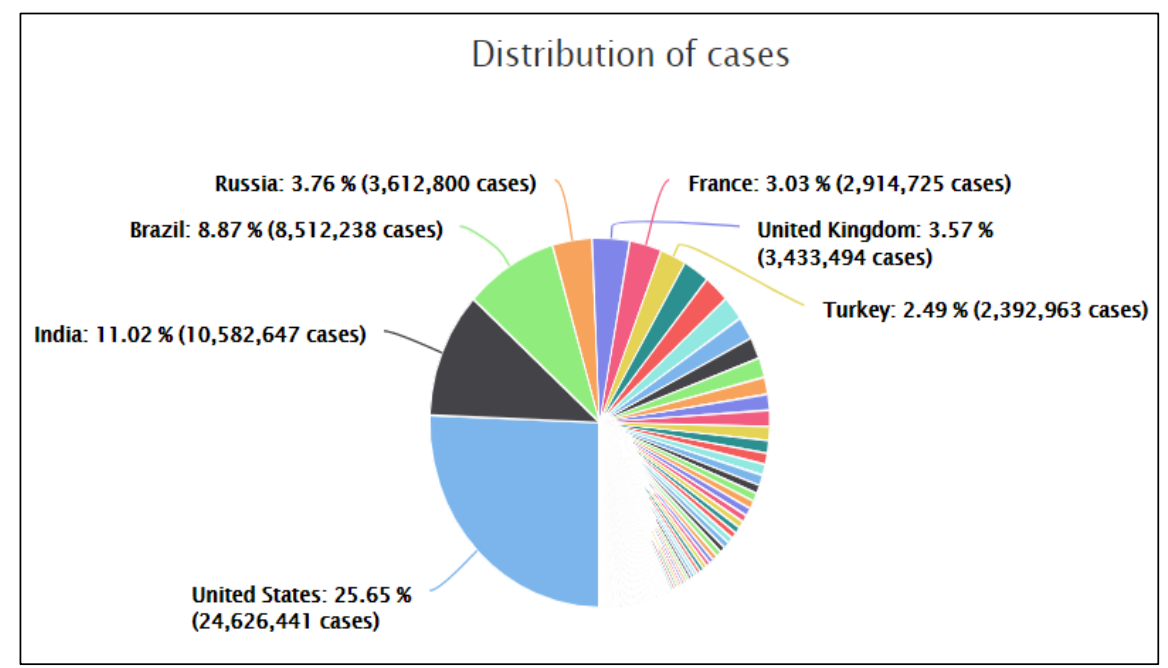

Fig. 13: Country-wise 2019-nCoV cases distribution (Outside china), 17 th January 2021 [109]

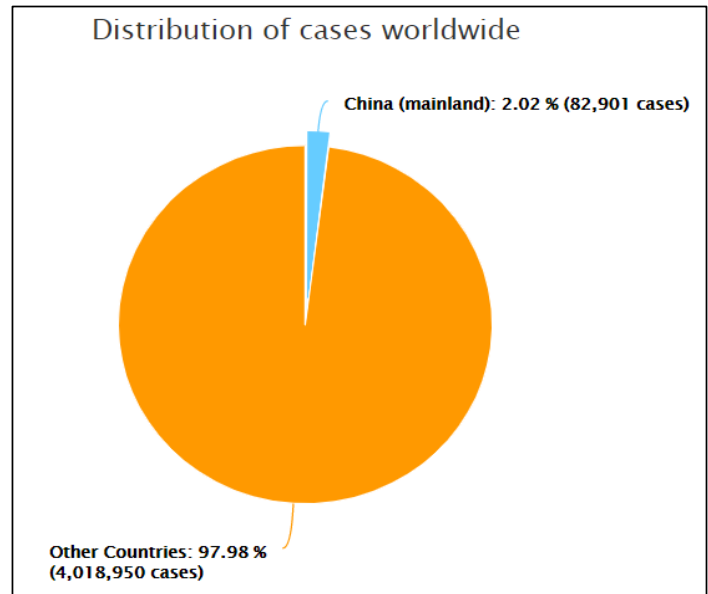

Fig. 14: Country-wise 2019-nCoV cases distribution (Worldwide) [108] 


\section{(b) Total death cases (globally)}

Fig. 15 describes the total confirmed death cases globally due to the 2019-nCoV pandemic. Total lives are claimed by this deadly novel coronavirus from $23^{\text {rd }}$ January 2020 until $17^{\text {th }}$ January 2021 and were recorded as 2,149,311. The maximum rate of change in aggregate death was observed during its starting period on $24^{\text {th }}$ January 2020 with an aggregate of $64 \%$. After $07^{\text {th }}$ April 2020, the rate change in absolute death was decreased gradually $(<10 \%)$ and later on $24^{\text {th }}$ April 2020 onwards almost consistent ranging from $1 \%$ to $3 \%$.

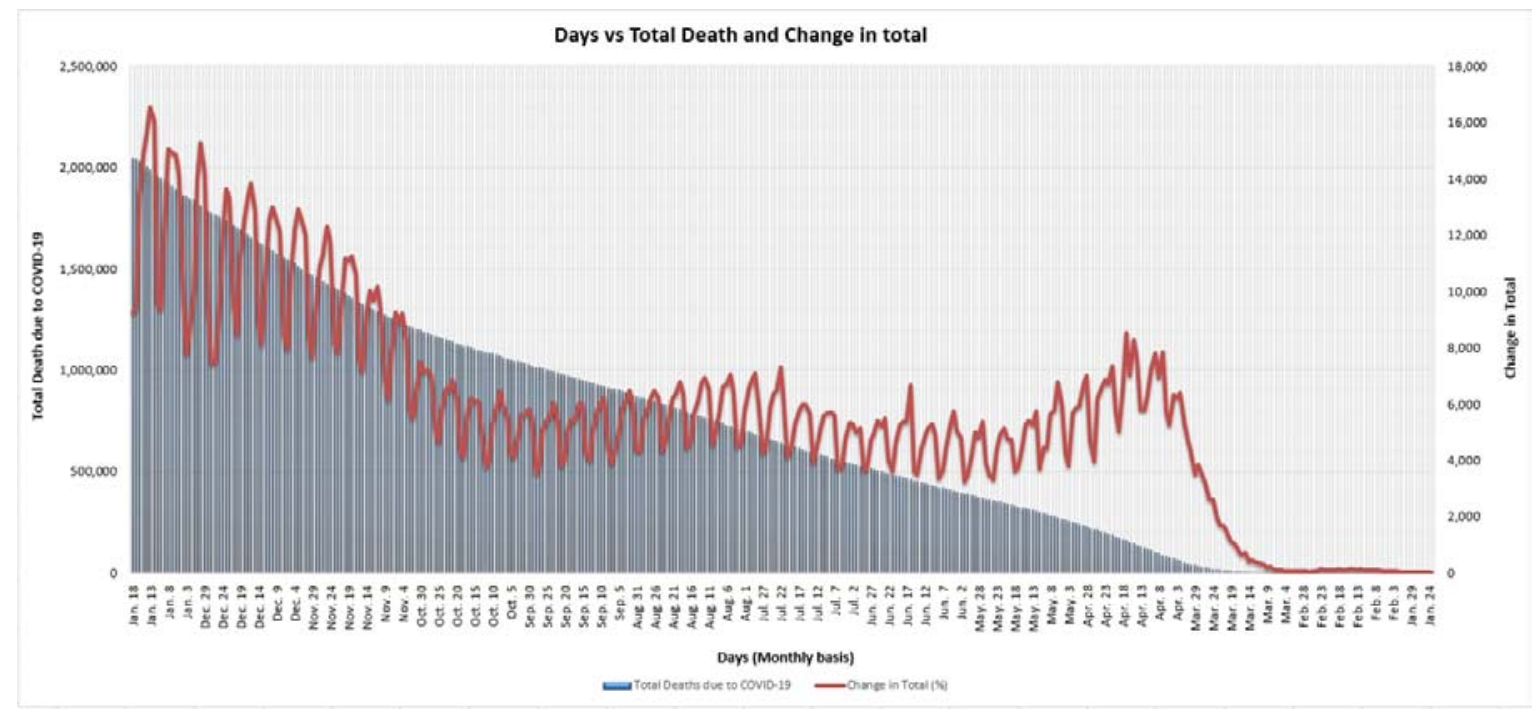

Fig. 15: 2019-nCoV total death globally and change in total (\%), 17 th $^{\text {January }} 2021$

\section{(c) Country affected due to COVID-19 (Top 60's)}

Fig. 16 refers to the top 60 countries in the world which are most severely affected by 2019 -nCoV. It reflects the cumulative number of cases and deaths in each country during the pandemic. The world's most powerful country has been harshly affected having the highest number of COVID-19 cases $(25,862,957)$ and death $(431,407)$ respectively as reported by Worldometer on $25^{\text {th }}$ January 2021 [97].
The second-highest number of confirmed cases was reported in India $(10,677,710)$ and the death toll in Brazil $(217,712)$. The worst death toll and cases reported in different countries of Europe, Asia, North America, South America, Africa, and Oceania continents are shown in table 10 . In China, from where the outbreak story was started, reported 89,115 cases and 4,635 death. Among the Asian countries, India lies in the second position next to the USA with total cases of $10,677,710$ and 153,624 deaths.

Table 10: Most severely affected countries in different continents, $2^{\text {th }}$ January 2021

\begin{tabular}{|c|c|c|}
\hline \multirow[t]{2}{*}{ Continents } & \multicolumn{2}{|l|}{ Country most severely affected } \\
\hline & Total cases & Total deaths \\
\hline Europe & Russia (3738690), UK (3669657) & UK $(98,531)$, Italy $(85,881)$ \\
\hline Asia & India $(10677710)$, Turkey $(2,435,247)$ & India $(1,53624)$, Iran $(57,481)$ \\
\hline North America & USA $(25,862,957)$, Mexico $(1,763,219)$ & USA (431407), Mexico(149614) \\
\hline South America & Brazil $(8,872,964)$, Colombia $(2,027,746)$ & Brazil $(217,712)$, Colombia $(51,747)$ \\
\hline Africa & South Africa $(1,417,537)$, Egypt $(162,486)$ & South Africa $(41,117)$, Egypt $(9,012)$ \\
\hline Oceania & Australia $(28,777)$, French Polynesia $(17,912)$ & Australia (909), French Polynesia (129) \\
\hline
\end{tabular}

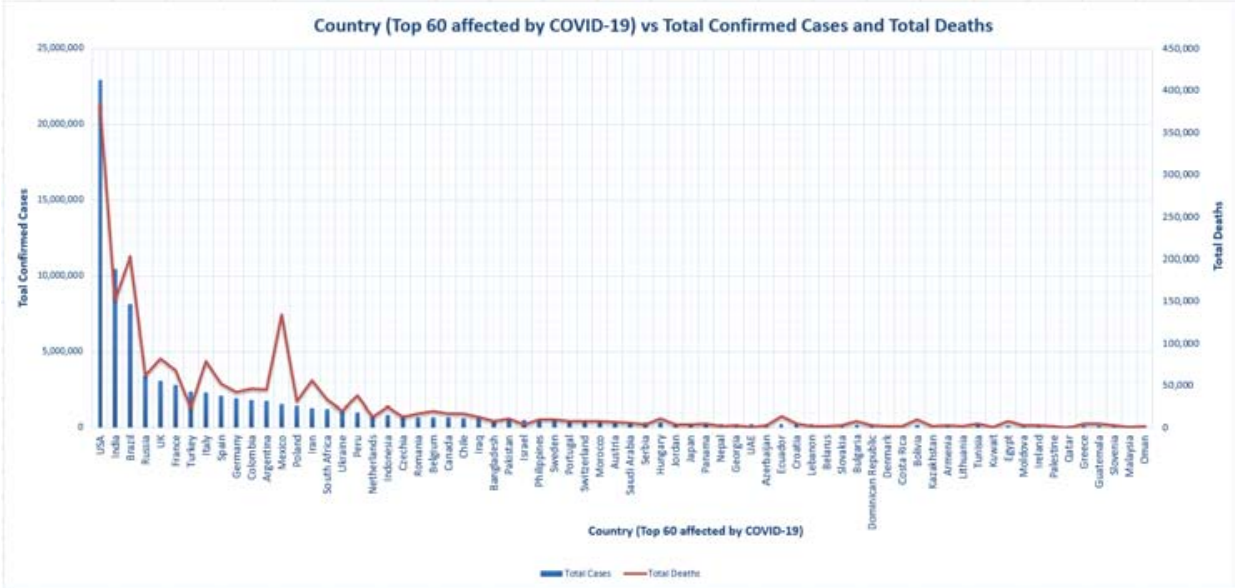

Fig. 16: 2019-nCoV total cases and deaths country wise (arranged in descending order), $17^{\text {th }}$ January 2021 


\section{(d) Daily Cases Growth Factor (Globally)}

A growth factor is the factor of growth in which the quantity multiplies over time [110]. It expresses as:

Growth Factor $=$ New Cases $($ Present Day) $/$ New Cases (Previous Day)

A growth factor above 1 implies a rise, whereas between 0 and 1 is a symbol of the decrease and the number gradually becomes zero, whereas a growth factor above 1 continuously may be an indicator of exponential growth. For example, a quantity growing by $7 \%$ every period (daily) has a growth factor of 1.07[108]. Fig. 17 represents the growth factor curve for daily new cases worldwide from 24th January 2020 to 25th January 2021[111]. The observed peaks in the growth factor curves represent the hikes in daily-confirmed cases of COVID-19. The maximum rise in everyday cases growth factor was observed on 12th February 2020 with a peak value of 6.95 and a second maximum on 27th January 2020 with a peak value of 2.27 . On 25th January 2021, the daily cases have been recorded as less than 1 , i.e., 0.93 , which signifies the sign of decline in the rate of daily new cases. However, it has to be noted that the maximum rise in daily cases growth factor was observed during the starting period of the pandemic.

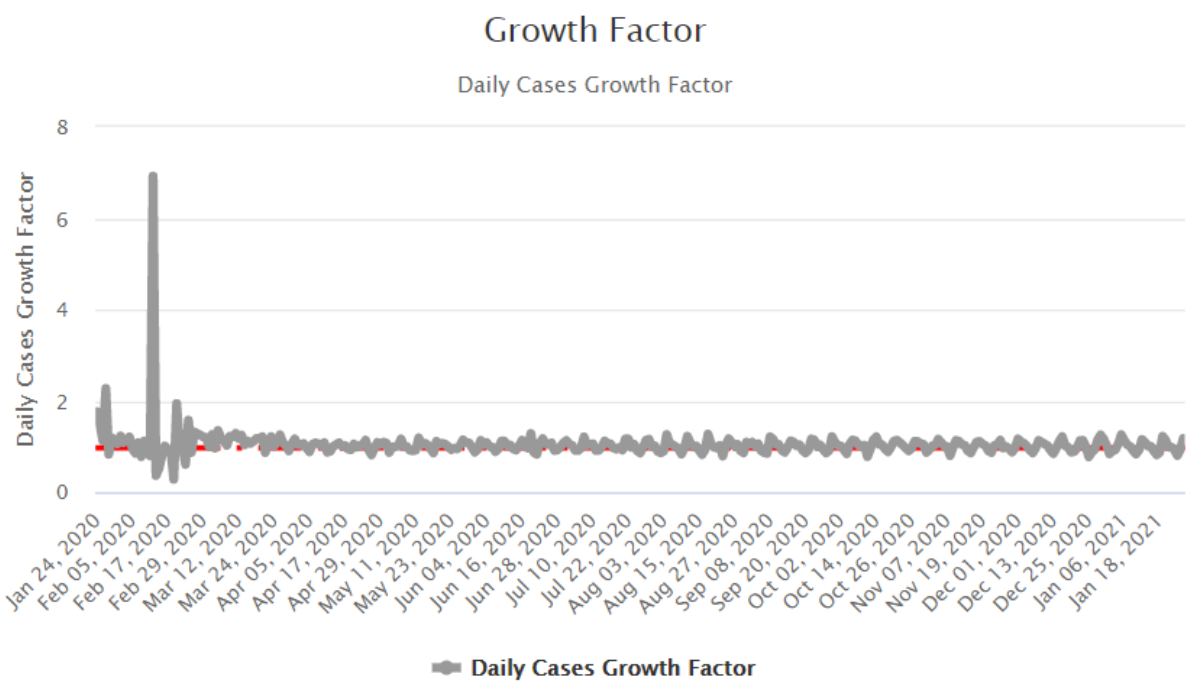

Fig. 17: 2019-nCoV worldwide daily cases growth factor, 25 January 2021 [111]

\section{COVID-19 vaccination: An Overview}

The worldwide endeavor to set up a safe and effective COVID-19 vaccine is bearing fruit. WHO started work on COVID-19 vaccine research and development in February 2020, after consultation with multiple scientists and health experts [112]. The COVID-19 Vaccines Global Access (COVAX) facility was established by WHO in collaboration with the ACT-Accelerator vaccine partners the Coalition for Epidemic Preparedness Innovations (CEPI) and Gavi, the Vaccine Alliance [113]. The motive behind the establishment of COVAX is to bring the nation together, regardless of their income level, to ensure the procurement and equitable distribution of COVID-19 vaccines. Since the vaccine resources remain scarce, therefore, immunization programs have to prioritize certain age groups over others before expanding their distribution to all population groups [114]. The prioritized groups include:

(i) Frontline Workers in Health and social care settings

(ii) People having age $\geq 65$

(iii) People having age $<65$, having underlying health conditions leading to the death risk.

As determined by the WHO Strategic Advisory Group of Experts the immunization will be carried out for prioritized groups in different phases as described in table 11 .

Table 11: Phases of immunization [113]

\begin{tabular}{ll}
\hline Phases & Description \\
\hline Phase I & $\begin{array}{l}\text { Doses will be made available to participating countries simultaneously until they cover approximately 20\% } \\
\text { of the population. In most countries, the firstly prioritized group includes the frontline workers in health and } \\
\text { social care settings. } \\
\text { The pace at which countries will receive additional dosage would be analyzed by risk assessment at any } \\
\text { given time. Consideration will be based on threat, vulnerability (health care system and population). } \\
\text { Special consideration to those countries that may suddenly face a major outbreak of the disease throughout } \\
\text { the allocation process. } \\
\text { In addition to the vaccine allocation in Phase I and II, some doses of vaccines will be reserved as part of the } \\
\text { humanitarian Buffer } \\
\text { A small buffer of up to 5\% of the total number of available dosages will be considered as a buffer for the } \\
\text { vulnerable population which may include Refugees, Migrants, Detainees, Asylum seekers, and workers } \\
\text { involved in these settings. }\end{array}$ \\
\end{tabular}

The major WHO vaccinations and immunization consulting group are the Strategic Expert Advisory Group (SAGE). The production, development, and distribution of vaccines are taken care of by SAGE providing independent experts advice and recommendations towards safe and equitable distribution of the approved vaccines
$[114,115]$. A handful of vaccines now have been authorized around the globe, and many more remain in progress. Table 12 presents the details of vaccines that have achieved authorization and approval for vaccination. Table 13 is the list of vaccines in Phase 1-3 clinical trials [112]. 
Table 12: List of approved/authorized vaccines

\begin{tabular}{|c|c|c|c|c|c|}
\hline Name & $\begin{array}{l}\text { Vaccine } \\
\text { type }\end{array}$ & Primary developer & $\begin{array}{l}\text { Origin } \\
\text { country }\end{array}$ & Approval & References \\
\hline $\begin{array}{l}\text { Comirnaty } \\
\text { (BNT162b2) }\end{array}$ & $\begin{array}{l}\text { mRNA- } \\
\text { based } \\
\text { vaccine }\end{array}$ & $\begin{array}{l}\text { Pfizer, BioNTech; Fosun } \\
\text { Pharma }\end{array}$ & Multinational & $\begin{array}{l}\text { United Kingdom, Bahrain, Canada, Mexico, US, } \\
\text { Singapore, Costa Rica, Ecuador, Jordan, } \\
\text { Panama, Chile, Oman, Saudi Arabia, Argentina, } \\
\text { Switzerland, Kuwait, EU, Philippines, Pakistan, } \\
\text { Colombia, Iraq, Israel, Qatar, Singapore, United } \\
\text { Arab Emirates, Faroe Islands, Greenland, } \\
\text { Iceland, Malaysia, Norway, Serbia }\end{array}$ & [112] \\
\hline $\begin{array}{l}\text { Moderna } \\
\text { COVID-19 Vaccine } \\
\text { (mRNA-1273) }\end{array}$ & $\begin{array}{l}\text { mRNA- } \\
\text { based } \\
\text { vaccine }\end{array}$ & Moderna, BARDA, NIAID & US & $\begin{array}{l}\text { Canada, Israel, Saudi Arabia, Switzerland, } \\
\text { United Kingdom, United States, EU, Faroe } \\
\text { Islands, Greenland, Iceland, Norway }\end{array}$ & [112] \\
\hline CoronaVac & $\begin{array}{l}\text { Inactivated } \\
\text { vaccine }\end{array}$ & Sinovac & China & China, Bolivia, Turkey, Indonesia, Brazil & [112] \\
\hline $\begin{array}{l}\text { COVID-19 Vaccine } \\
\text { AstraZeneca } \\
\text { (AZD1222) }\end{array}$ & $\begin{array}{l}\text { Adenovirus } \\
\text { vaccine }\end{array}$ & BARDA, OWS & UK & $\begin{array}{l}\text { UK, Argentina, El Salvador, Dominican } \\
\text { Republic, India, Bangladesh, Mexico, Nepal, } \\
\text { Pakistan, Brazil, Saudi Arabia, Iraq, Hungary, } \\
\text { Thailand }\end{array}$ & [112] \\
\hline Sputnik V & $\begin{array}{l}\text { Non- } \\
\text { replicating } \\
\text { viral vector }\end{array}$ & $\begin{array}{l}\text { Gamaleya Research } \\
\text { Institute, Acellena Contract } \\
\text { Drug Research and } \\
\text { Development }\end{array}$ & Russia & $\begin{array}{l}\text { Russia, Belarus, Argentina, Guinea, Bolivia, } \\
\text { Algeria, Palestine, Venezuela, Paraguay, } \\
\text { Turkmenistan, Hungary, UAE, Serbia }\end{array}$ & [112] \\
\hline BBIBP-CorV & $\begin{array}{l}\text { Inactivated } \\
\text { vaccine }\end{array}$ & $\begin{array}{l}\text { Beijing Institute of } \\
\text { Biological Products; China } \\
\text { National Pharmaceutical } \\
\text { Group (Sinopharm) }\end{array}$ & China & $\begin{array}{l}\text { China, Bahrain, United Arab Emirates, Egypt, } \\
\text { Jordan, Iraq, Pakistan, Serbia }\end{array}$ & [112] \\
\hline EpiVacCorona & $\begin{array}{l}\text { Peptide } \\
\text { vaccine }\end{array}$ & $\begin{array}{l}\text { Federal Budgetary Research } \\
\text { Institution State Research } \\
\text { Center of Virology and } \\
\text { Biotechnology }\end{array}$ & Russia & Russia & [112] \\
\hline Covaxin & $\begin{array}{l}\text { Inactivated } \\
\text { vaccine }\end{array}$ & Bharat Biotech, ICMR & India & India & [112] \\
\hline
\end{tabular}

Table 13: List of approved/authorized vaccines in trial phase II and III

\begin{tabular}{|c|c|c|c|c|c|}
\hline Candidate & Mechanism & Sponsor & $\begin{array}{l}\text { Trial } \\
\text { phase }\end{array}$ & Institution & References \\
\hline $\begin{array}{l}\text { JNJ-78436735 } \\
\text { (formerly Ad26. } \\
\text { COV2.S) }\end{array}$ & $\begin{array}{l}\text { Non-replicating viral } \\
\text { vector }\end{array}$ & Johnson and Johnson & Phase III & Johnson and Johnson & [112] \\
\hline NVX-CoV2373 & Nanoparticle vaccine & Novavax & Phase III & Novavax & [112] \\
\hline $\begin{array}{l}\text { Convidicea } \\
\text { (Ad5-nCoV) }\end{array}$ & $\begin{array}{l}\text { Recombinant vaccine } \\
\text { (adenovirus type } 5 \\
\text { vector) }\end{array}$ & CanSino Biologics & Phase III & Tongji Hospital; Wuhan, China & [112] \\
\hline $\begin{array}{l}\text { Bacillus } \\
\text { Calmette- } \\
\text { Guerin (BCG) } \\
\text { vaccine }\end{array}$ & Live-attenuated vaccine & $\begin{array}{l}\text { University of Melbourne and } \\
\text { Murdoch Children's Research } \\
\text { Institute; Radboud University } \\
\text { Medical Center; Faustman Lab } \\
\text { at Massachusetts General } \\
\text { Hospital }\end{array}$ & $\begin{array}{l}\text { Phase } \\
\text { II/III }\end{array}$ & $\begin{array}{l}\text { University of Melbourne and Murdoch } \\
\text { Children's Research } \\
\text { Institute; Radboud University Medical } \\
\text { Center; Faustman Lab at } \\
\text { Massachusetts General Hospital }\end{array}$ & [112] \\
\hline INO-4800 & DNA vaccine (plasmid) & Inovio Pharmaceuticals & $\begin{array}{l}\text { Phase } \\
\text { II/III }\end{array}$ & $\begin{array}{l}\text { Center for Pharmaceutical Research, } \\
\text { Kansas City. Mo.; University of } \\
\text { Pennsylvania, Philadelphia }\end{array}$ & [112] \\
\hline VIR-7831 & $\begin{array}{l}\text { Plant-based adjuvant } \\
\text { vaccine }\end{array}$ & Medicago; GSK; Dynavax & $\begin{array}{l}\text { Phase } \\
\text { II/III }\end{array}$ & Medicago & [112] \\
\hline $\mathrm{CVnCoV}$ & mRNA-based vaccine & CureVac & $\begin{array}{l}\text { Phase } \\
\text { II/III }\end{array}$ & CureVac & [112] \\
\hline ZyCoV-D & DNA vaccine (plasmid) & Zydus Cadila & Phase II & Zydus Cadila & [112] \\
\hline BNT162 & mRNA-based vaccine & Pfizer, BioNTech & $\begin{array}{l}\text { Phase } \\
\text { I/II/III }\end{array}$ & $\begin{array}{l}\text { Multiple study sites in Europe, North } \\
\text { America and China }\end{array}$ & [112] \\
\hline IIBR-100 & $\begin{array}{l}\text { Recombinant vesicular } \\
\text { stomatitis virus (rVSV) } \\
\text { vaccine }\end{array}$ & & $\begin{array}{l}\text { Phase } \\
\text { I/II }\end{array}$ & $\begin{array}{l}\text { Hadassah Medical Center; Sheba } \\
\text { Medical Center Hospital }\end{array}$ & [112] \\
\hline
\end{tabular}

\section{CONCLUSION}

The growing number of cases involving 2019-nCoV and the global distribution of the disease raises questions about the future mechanism of the disease. The 2019-nCoV epidemic has prompted numerous nations to take unprecedented steps to curb the dissemination of the virus in the public health system. Such as India implemented nationwide lockdown-Phase I on 24th March 2020 for 21days which needed to be further extended to phase II from $15^{\text {th }}$ April 2020 to 03rd May 2020 and then to phase III commenced from $04^{\text {th }}$ May till $17^{\text {th }}$ May 2020. Similarly, execution of Movement Control Order (MCO) in Malaysia from $18^{\text {th }}$ March 2020 to $09^{\text {th }}$ June 
2020. To prevent further disease spread, travel controls were introduced followed by the suspension of transport by airports, train stops, and roads. However, COVID-19 has already crossed the outbreak of SARS by the number of affected cases and death tolls. Taking account of the financial effect, global production was anticipated to grow modestly by $2.5 \%$ in 2020 before the COVID-19 outbreak according to the World Economic Situation and Prospects for 2020. However, after the COVID-19 global pandemic, World Economic Forecasting Model, has estimated the worst scenario for global growth in 2020, i.e., the global output would contract by almost $1 \%$ with an economical effect of much larger than $\$ 360$ billion [39].

Besides, details of epidemiological properties, including animal resource recognition and risk factor, are vital to the prevention of COVID-19 outbreaks [115]. The middle hosts with the disease are important not only to diagnose the existing epidemic but also to avoid a potential outbreak. Education programs should be put in place to encourage public precautions, including regular hand washes, cough labels, and the use of personal wearing facial masks and hand sanitizers during public visits. Public safety initiatives should be deployed in this area. Active screening must be carried out for new cases and carefully monitoring their interactions [114]. To improve detection efficiency, hospitals and clinics should be armed with diagnostic kits. Beyond supportive care, the chase for COVID-19 vaccination is equally vital. Although no registered COVID-19 treatment or vaccine is available at this time. Many clinical trials have already been initiated and many on their way for testing [117]. For example, Oxford University has begun the Phase-1 Human Clinical Trial of its vaccine ChAdOx1 nCoV-19 against COVID-19 on 23rd April 2020 [118]. On 02nd April 2020, the University of Pittsburgh School of Medicine also announces the clinical trial of the developed vaccine, PittCoVacc (Pittsburgh Coronavirus Vaccine) against the COVID-19 pandemic [119]. Similarly, Novavax, Inc., a clinical stage biotechnology company, announced the first in a human trial of their vaccine NVX-CoV2373 in mid-May [120]. Recent work on Bacillus Calmette-Guerin (BCG-CORONA) vaccine to fight against COVID-19 disease has been carried out in conjunction with the University of Melbourne's Murdoch Children's Research Institute (Australia), the Radboud University Medical Center in The Netherlands and Massachusetts General Hospital (The United States) Faustman Lab. BCG was already developed vaccine against tuberculosis. However research studies have shown its potential in preventing acute respiratory tract infections. Based on this hypothesis, the efficacy BCG vaccination is under study to evaluate its potential for protection against the severity of COVID-19 infection $[119,120]$.

Currently, Zhang and Liu [123] proposed that the status of COVID-19 affected patients to be reviewed before monitoring general therapies for their nutritional presence (Vitamins A, B, C, D, E, Selenium, Zinc, and Iron) as it could lead to the treatment of COVID-19 in the emergency medical assistance. Even the possible antidote to this new coronavirus can be regarded as unique antivirals, which were highly successful in treating SARS-CoV and MERS-CoV [124]. The development of the SARS vaccine has already attracted the attention of many scientists for the treatment of COVID-19 illness. SARS-CoV is identical to the avian infectious bronchitis virus (A-IBV) and both belong to the same family [125]. Bijlenga et al. [126] proposed using the IBV (strains $\mathrm{H}$ ) avian live virus vaccine in 2005 for the treatment of SARS. Therefore, the IBV vaccine approach may also be effectively considered as a treatment against COVID-19 infection. However, preliminary tests on animals should be taken into consideration before the startup. In comparison, passive immunotherapy can be called Convalescent Plasma [127]. This therapy is frequently adopted where no special vaccines or medicines are accessible for new pathogens infected with viruses, as with the latest pandemic scenario. Arabi et al. [128] tested the efficacy and therapeutic safety of the convalescent plasma treatment in chronically ill patients with MERS. Depending on their results, convalescent plasma had an immunotherapy capacity to treat an infection of MERS-CoV. In the treatment of SARS-CoV patients, convalescent plasma retrieved from SARS patients was also stated clinically helpful [129, 130]. Prominently, WHO has also suggested the use of convalescent plasma or serum when vaccines or antiviral drugs are not available for an emerging virus such as a 2019-nCoV [123]. Lastly, as children are often infected by SARS-CoV, the RNA-virus vaccine program helps them in treatment against this infection. Vaccines associated with RNA viruses include measles, polio, Japan's encephalitis virus, influenza virus and vaccine associated with rabies [131]. Therefore, RNA-virus vaccines may be considered as a promising alternative towards COVID-19 treatment and prevent transmission between people by immunizing health workers and non-infected people.

As a concluding remark, as there are many unknowns yet to be explored with SARS-CoV-2 and the whole world is currently engaged in a challenging battle with an unseen enemy-COVID-19, the time to act is now. As stated in table 12 and 13 about the approved vaccines and vaccines under clinical trials, which shows that the whole world has united in the combat against COVID-19 and come up with a united effort to defeat and win this combat making the world as free, happier, healthier and livable place as it was before COVID-19.

\section{ACKNOWLEDGMENT}

The authors would like to acknowledge National Taiwan University of Science and Technology (NTUST), Taiwan and Rajkiya Engineering College, Ambedkar Nagar, India to facilitate this research study. The authors would also like to thank the reviewers for their valuable suggestion to enhance the manuscript of interests regarding the publication of this paper

\section{FUNDING}

The authors would like to thanks Rajkiya Engineering College, Ambedkar Nagar, Uttar Pradesh, India to facilitate this research study under the project Third Phase of Technical Education Quality Improvement Programme (TEQIP-III), a Government of India Project assisted by the World Bank.

\section{AUTHORS CONTRIBUTIONS}

Conceptualization, A. A; Methodology, A. A, and V. S. C; Investigation, A. A, and N. A; Writing-Original Draft Preparation, A. A, and S. P. S; Writing-Review and Editing, A. A, N. A and S. P. S; Supervision, V. S. C and S. P.S.

\section{CONFLICTS OF INTERESTS}

The authors declare that they have no conflict of interest.

\section{REFERENCES}

1. Bansal M, Walia MK. COVID 19-an overview on epidemiology, symptoms, prevention, management, treatment and role of health workers. Int J Appl Pharm 2020;12:36-41.

2. Diseases E, Tracker D, Careers E. In focus coronavirus [Internet]. Infectious Diseases Hub; 2020. Available from: https://www.id-hub.com/2020/03/17/in-focus-coronavirus/

3. Weiss SR, Navas Martin S. Coronavirus pathogenesis and the emerging pathogen severe acute respiratory syndrome coronavirus. Microbiol Mol Biol Rev 2005;69:635-64.

4. Liu C, Zhou Q, Li Y, Garner LV, Watkins SP, Carter LJ, et al. Research and development on therapeutic agents and vaccines for COVID-19 and related human coronavirus diseases. ACS Cent Sci 2020;6:315-31.

5. Niharika D, Niharika B, Aishwarya T, Nikitha A, Butool R, Ibrahim $\mathrm{M}$, et al. Coronavirus-a virus in learning. Int J Curr Pharm Res 2020;12:7-10.

6. Centers for Disease Control and Prevention. Public Health Image Library (PHIL); 2020. Available from: https://phil.cdc.gov/Details.aspx?pid=23313 [Last accessed on 01 Jan 2021]

7. Du L, He Y, Zhou Y, Liu S, Zheng BJ. The spike protein of SARS$\mathrm{CoV}$-a target for vaccine and therapeutic development. Nat Rev Microbiol 2009;7:226-36.

8. Cavanagh D. Coronavirus avian infectious bronchitis virus. Vet Res 2007;38:281-97.

9. Ismail AMM, Tang Y, Saif YM. Pathogenicity of turkey coronavirus in turkeys and chickens. Avian Dis 2003;47:515-22.

10. Su S, Wong G, Shi W, Liu J, Lai ACK, Zhou J, et al. Epidemiology, genetic recombination, and pathogenesis of coronaviruses. Trends Microbiol 2016;24:490-502. 
11. Zhou P, Fan H, Lan T, Yang X, Shi W, Zhang W, et al. Fatal swine acute diarrhoea syndrome caused by an HKU2-related coronavirus of bat origin. Nature 2018;556:255-8.

12. Gallagher TM, Buchmeier MJ. Coronavirus spike proteins in viral entry and pathogenesis. Virology 2001;279:371-4.

13. Tyrrell DAJ, Bynoe ML, Obst Drcog. Cultivation of novel type of common-cold virus in organ cultures. Br Med J 1965;1:1467-70.

14. Wang C, Horby PW, Hayden FG, Gao GF. A novel coronavirus outbreak of global health concern. Lancet 2020;395:470-3.

15. Zhu N, Zhang D, Wang W, Li X, Yang B, Song J, et al. A novel coronavirus from patients with pneumonia in China, 2019. New Engl J Med 2020;382:727-33.

16. Lu R, Zhao X, Li J, Niu P, Yang B, Wu H, et al. Articles genomic characterisation and epidemiology of 2019 novel coronavirus: implications for virus origins and receptor binding. Lancet 2020;395:565-74.

17. Peiris JSM, Guan Y, Yuen KY. Severe acute respiratory syndrome. Nat Med 2004;10:88-97.

18. Peiris JSM, Lai ST, Poon LLM, Guan Y, Yam LYC, Lim W, et al. Coronavirus as a possible cause of severe acute respiratory syndrome. Lancet 2003;361:1319-25.

19. Chan Yeung M, Xu R. SARS: epidemiology. Respirology 2003;8:9-14.

20. Raj VS, Osterhaus ADME, Fouchier RAM, Haagmans BL. MERS: emergence of a novel human coronavirus. Curr Opin Virol 2014;5:58-62.

21. Lee J, Chowell G, Jung E. A dynamic compartmental model for the Middle East respiratory syndrome outbreak in the republic of korea: a retrospective analysis on control interventions and superspreading events. J Theor Biol 2016;408:118-26.

22. Schoeman D, Fielding BC. Coronavirus envelope protein: current knowledge. Virol J 2019;16:1-22.

23. StatNano. Coronavirus Disease 2019 (COVID-19) Outbreak. StatNano; $2020 . \quad$ Available from: https://statnano.com/coronavirus-disease-outbreak-covid-19 [Last accessed on 01 Jan 2021]

24. World Health Organization. WHO Novel Coronavirus-Japan (ex-China). World Health Organization; 2020. Available from: https://www.who.int/csr/don/16-january-2020-novelcoronavirus-japan-ex-china/en/ [Last accessed on 01 Jan 2021]

25. World Health Organization. Novel Coronavirus (2019-nCoV) SITUATION REPORT-1; 2020. Available from: https://www.who.int/docs/defaultsource/coronaviruse/situation-reports/20200121-sitrep-12019-ncov.pdf [Last accessed on 01 Jan 2021]

26. World Health Organization. Novel Coronavirus (2019-nCoV); 2020. Available from: https://www.who.int/docs/defaultsource/coronaviruse/situation-reports/20200124-sitrep-42019-ncov.pdf?sfvrsn=9272d086_8 [Last accessed on 01 Jan 2021]

27. Gralinski LE, Menachery VD. Return of the coronavirus: 2019nCoV. Viruses 2020;12:1-8.

28. World Health Organization. WHO Timeline-COVID-19 this statement is updated on an ongoing. World Health Organization; 2020. p. 1-4. Available from: https://www.who.int/news-room/detail/08-04-2020-whotimeline---covid-19 [Last accessed on 01 Jan 2021]

29. World Health Organization. Coronavirus Disease 2019 (COVID19) Situation Report-11. World Health Organization; 2020. p. 1-15. Available from: https://www.who.int/docs/defaultsource/searo/timor-leste/17-02-2020-tls-sitrep-11-ncov-eng. pdf?sfvrsn=d7f73101_2 [Last accessed on 01 Jan 2021]

30. World Health Organization. WHO Statement regarding cluster of pneumonia cases in Wuhan, China. World Health Organization; 2020. p. 1-2. Available from: https://www.who.int/china/news/detail/09-01-2020-whostatement-regarding-cluster-of-pneumonia-cases-in-wuhanchina [Last accessed on 01 Jan 2021]

31. Gorbalenya AE, Baker SC, Baric RS, Groot RJ De, Gulyaeva AA, Haagmans BL, et al. Severe acute respiratory syndrome-related coronavirus: the species and its viruses-a statement of the coronavirus study group. bioRxiv. 2020:1-15. https://doi.org/10.1101/2020.02.07.937862
32. Ji W, Wang W, Zhao X, Zai J, Li X. Homologous recombination within the spike glycoprotein of the newly identified coronavirus may boost cross-species transmission from snake to human. J Med Virol 2020;92:433-40.

33. Cyranoski D. Did pangolins spread the China coronavirus to people? Nature 2020. https://doi.org/10.1038/d41586-02000364-2

34. Wang W, Tang J, Wei F. Updated understanding of the outbreak of 2019 novel coronavirus (2019-nCoV) in Wuhan, China. J Med Virol 2020;92:441-7.

35. Enserink M. Update A bit chaotic. Christening of new coronavirus and its disease name create confusion. Science 2020. p. 1-4. Available from: https://www.sciencemag.org/news/2020/02/bit-chaoticchristening-new-coronavirus-and-its-disease-name-createconfusion [Last accessed on 01 Jan 2021]

36. World Health Organization. Coronavirus disease 2019 (COVID19) Situation Report-48. World Health Organization; 2020. p. 1-8. Available from: https://www.who.int/docs/defaultsource/coronaviruse/situation-reports/20200308-sitrep-48covid-19.pdf?sfvrsn=16f7ccef_4 [Last accessed on 01 Jan 2021]

37. Broughton JP, Deng X, Yu G, Fasching CL, Servellita V, Singh J, et al. CRISPR-Cas12-based detection of SARS-CoV-2. Nat Biotechnol 2020;38:870-4.

38. World Health Organization. Laboratory testing for coronavirus disease 2019 (COVID-19) in suspected human cases. World Health Organization; 2020. p. 1-10. Available from: https://apps.who.int/iris/bitstream/handle/10665/331329/ WHO-COVID-19-laboratory-2020.4-eng.pdf [Last accessed on 01 Jan 2021]

39. Nguyen T, Duong Bang D, Wolff A. 2019 Novel coronavirus disease (covid-19): paving the road for rapid detection and point-of-care diagnostics. Micromachines. 2020;11:306.

40. Gorbalenya AE, Baker SC, Baric RS, Groot RJ de, Drosten C, Gulyaeva AA, et al. The species severe acute respiratory syndrome-related coronavirus: classifying 2019-nCoV and naming it SARS-CoV-2. Nat Microbiol 2020;5:536-44.

41. Kupferschmidt $\mathrm{K}$, Cohen J. Will novel virus go pandemic or be contained? Science 2020;367:610-1.

42. Wu F, Zhao S, Yu B, Chen YM, Wang W, Song ZG, Hu Y, et al. A new coronavirus associated with human respiratory disease in China. Nature 2020;579:1-8.

43. Adnan N, Nordin SM. How COVID 19 effect Malaysian paddy industry? Adoption of green fertilizer a potential resolution. Environ Dev Sustain; 2020. https://doi.org/10.1007/s10668020-00978-6

44. Fehr AR, Channapannavar R, Perlman S. Middle east respiratory syndrome: Emergence of a pathogenic human coronavirus. Annu Rev Med 2017;68:387-99.

45. Zhou P, Yang X, Wang X, Hu B, Zhang L, Zhang W, et al. A pneumonia outbreak associated with a new coronavirus of probable bat origin. Nature 2020;588:1-10.

46. Centers for Disease Control and Prevention. Interim Guidelines for Collecting, Handling, and Testing Clinical Specimens from Persons for Coronavirus Disease 2019 (COVID-19). Centers for Disease Control and Prevention; 2020. Available from: https://www.cdc.gov/coronavirus/2019-ncov/lab/guidelinesclinical-specimens.html [Last accessed on 01 Jan 2021]

47. Holshue ML, DeBolt C, Lindquist S, Lofy KH, Wiesman J, Bruce H, Spitters C, et al. First case of 2019 novel coronavirus in the United States. N Engl J Med 2020;382:1177-9.

48. Zou L, Ruan F, Huang M, Liang L, Huang H, Hong Z, et al. SARS$\mathrm{CoV}-2$ viral load in upper respiratory specimens of infected patients. N Engl J Med 2020;382:1-3.

49. Guan W, Ni Z, Hu Y, Liang W, Ou C, He J, et al. Clinical characteristics of 2019 novel coronavirus infection in China medRxiv; 2020. Available from: https://www.medrxiv.org/ content/early/2020/02/09/2020.02.06.20020974 [Last accessed on 01 Jan 2021].

50. Udugama B, Kadhiresan P, Kozlowski HN, Malekjahani A, Osborne M, Li VYC, et al. Diagnosing COVID-19: the disease and tools for detection. ACS Nano 2020;14:3822-35.

51. World Health Organization. Novel Coronavirus (2019-nCoV) situation REPORT-4; 2020. Available from: 
https://www.who.int/docs/defaultsource/coronaviruse/situation-reports/20200124-sitrep-4-2019ncov.pdf?sfvrsn=9272d086_8 [Last accessed on 01 Jan 2021]

52. Li X, Zai J, Wang X, Li Y. Potential of large 'first generation' human-to-human transmission of 2019-nCoV. J Med Virol 2020;92:448-54.

53. Doremalen N van, Bushmaker T, Morris DH, Holbrook MG, Amandine Gamble, Brandi N Williamson, et al. Aerosol and surface stability of SARS-CoV-2 as compared with SARS-CoV-1. N Engl J Med 2020;382:1564-7.

54. Wadhwa P. Molecular docking study of novel covid-19 protease with current clinical management agents. Asian J Pharm Clin Res 2020;13:37-9.

55. Centers for Disease Control and Prevention. How COVID-19 Spreads; Vol. May. Centers for Disease Control and Prevention; $2020 . \quad$ Available from: https://www.cdc.gov/coronavirus/2019-ncov/faq.html\#HowCOVID-19-spreads [Last accessed on 01 Jan 2021]

56. Rothe C, Schunk M, Sothmann P, Bretzel G, Froeschl G Wallrauch C, et al. Transmission of 2019-nCoV infection from an asymptomatic contact in germany. $\mathrm{N}$ Engl J Med 2020;383:970-1.

57. Phan LT, Nguyen TV, Luong QC, Nguyen TV, Le HQ, Nguyen TT, et al. Importation and human-to-human transmission of a novel coronavirus in vietnam. $\mathrm{N}$ Engl J Med. 2020;382:872-4.

58. Worldometer. COVID-19 coronavirus pandemic, 28 January 2021. Worldometer; 2021. p. 1-22. Available from https://www.worldometers.info/coronavirus/ [Last accessed on 01 Jan 2021]

59. World Health Organization. COVID-19 Wly Epidemiological Update; 2021. Available from: https://www.who.int/docs/default-source/coronaviruse/ situation-reports/20201012-weekly-epi-update-9.pdf [Last accessed on 01 Jan 2021]

60. World Health Organization. Coronavirus disease 2019 (COVID19) Situation Report-48; 2020. Available from: https://www.who.int/docs/default-source/coronaviruse/ situation-reports /20200308-sitrep-48-covid19.pdf?sfvrsn=16f7ccef_4 [Last accessed on 01 Jan 2021]

61. Tang B, Bragazzi NL, Li Q, Tang S, Xiao Y, Wu J, et al. An updated estimation of the risk of transmission of the novel coronavirus (2019-nCov). Infect Dis Model 2020;5:248-55.

62. Cohen J, Kupferschmidt K. Strategies shift as coronavirus pandemic looms. Science 2020;367:962-3.

63. Regan H. Italy announces lockdown as global coronavirus cases surpass 105,000. CNN World; 2020. Available from: https://edition.cnn.com/2020/03/08/asia/coronavirus-covid19-update-intl-hnk/index.html [Last accessed on 01 Jan 2021]

64. Kobayashi T, Jung S, Linton NM, Kinoshita R, Hayashi $\mathrm{K}$, Miyama T, et al. Communicating the risk of death from novel coronavirus disease (COVID-19). J Clin Med 2020;9:1-7.

65. World Economic Forum. Key milestones in the spread of the coronavirus pandemic-a timeline. World Economic Forum; $2020 . \quad$ p. 1-15. Available from: https://www.weforum.org/agenda/2020/04/coronavirusspread-covid19-pandemic-timeline-milestones/ [Last accessed on 01 Jan 2021]

66. Mizumoto K, Kagaya K, Zarebski A, Chowell G. Estimating the asymptomatic proportion of coronavirus disease 2019 (COVID19) cases on board the diamond princess cruise ship, yokohama, Japan, 2020. Eurosurveillance 2020;25:1-5.

67. Stephen M Hahn. Coronavirus disease 2019 (COVID-19) update: foreign Inspections. U.S. Food and drug administration; 2020 Available from: https://www.fda.gov/news-events/pressannouncements/coronavirus-disease-2019-covid-19-updateforeign-inspections [Last accessed on 01 Jan 2021]

68. Guan W, Ni Z, Hu Y, Liang W, Ou C, He J, et al. Clinical characteristics of coronavirus disease 2019 in China. N Engl J Med 2020;382:1708-20.

69. Centers for Disease Control and Prevention. CDC 2019-nove coronavirus (2019-nCoV) real-time RT-PCR diagnostic panel, 2020. Available from: https://www.fda.gov/media/ 134922/download [Last accessed on 01 Jan 2021].
70. Yang W, Yan F. Patients with RT-PCR confirmed COVID-19 and normal chest CT. Radiology 2020;295:17-9.

71. World Health Organization. Coronavirus disease 2019 (COVID19) situation Report-179. Vol. 179. World Health Organization; 2020. Available from: https://www.who.int/docs/defaultsource/coronaviruse/situation-reports/20200717-covid-19sitrep-179.pdf?sfvrsn=2f1599fa_2 [Last accessed on 01 Jan 2021]

72. Read JM, Bridgen JRE, Cummings DAT, Ho A, Jewell CP. Novel coronavirus 2019-nCoV: early estimation of epidemiological parameters and epidemic predictions. medRxiv 2020:1-11. https://doi.org/10.1101/2020.01.23.20018549

73. Worldometer. COVID-19 coronavirus pandemic. Worldometer; 2020. Available from: https://www.worldometers.info/ coronavirus/ [Last accessed on 01 Jan 2021]

74. Majumder MS, Mandl KD. Early transmissibility assessment of a novel coronavirus in Wuhan, China. SSRN; 2020.

75. Imai N, Cori A, Dorigatti I, Baguelin M, Donnelly CA, Riley S, et al. Report 3: Transmissibility of 2019-nCoV; 2020

76. Wu JT, Leung K, Leung GM. Nowcasting and forecasting the potential domestic and international spread of the 2019-nCoV outbreak originating in Wuhan, China: a modelling study. Lancet 2020;6736:1-9.

77. World Health Organization. Statement on the meeting of the International Health Regulations (2005) Emergency Committee regarding the outbreak of novel coronavirus $(2019-\mathrm{nCoV})$. World Health Organization; 2020. Available from: https://www.who.int/news-room/detail/23-01-2020statement-on-the-meeting-of-the-international-healthregulations-(2005)-emergency-committee-regarding-theoutbreak-of-novel-coronavirus-(2019-ncov). [Last accessed on 01 Jan 2021]

78. Zhao S, Lin Q, Ran J, Musa SS, Yang G, Wang W, et al. Preliminary estimation of the basic reproduction number of novel coronavirus (2019-nCoV) in China, from 2019 to 2020: a data-driven analysis in the early phase of the outbreak. Int J Infect Dis 2020;92:214-7.

79. Manuel B, Richard K, Sarah T, H HH, F WA, A NR. 2019-novel coronavirus (2019-nCoV): estimating the case fatality rate-a word of caution. Swiss Med Wkly 2020:13. https://doi.org/10.4414/smw.2020.20203

80. World Health Organization. Novel coronavirus press conference at United Nations of Geneva; 2020. Available from: https://www.who.int/docs/defaultsource/coronaviruse/transcripts/who-audio-script-ncovrresser-unog-29jan2020.pdf?sfvrsn=a7158807_4 [Last accessed on 01 Jan 2021]

81. World Health Organization. WHO Director-General's opening remarks at the media briefing on COVID-19-3 March 2020; 2020. Available from: https://www.who.int/ $\mathrm{dg} /$ speeches/detail/who-director-general-s-opening-remarksat-the-media-briefing-on-covid-19---3-march-2020. [Last accessed on 01 Jan 2021]

82. Worldometer. Coronavirus (COVID-19) Mortality Rate. Worldometer; $2020 . \quad$ Available from: https://www.worldometers.info/coronavirus/coronavirusdeath-rate/\#ref-12. [Last accessed on 01 Jan 2021]

83. World Health Organization. Report of the WHO-China Joint Mission on Coronavirus Disease 2019 (COVID-19). Vol. 2019; 2020. Available from: https://www.who.int/docs/defaultsource/coronaviruse/who-china-joint-mission-on-covid-19final-report.pdf. [Last accessed on 01 Jan 2021]

84. National Health Commission (NHC) of the People's Republic of China. NHS Press Conference, Feb. 4 2020; 2020. Available from: http://www.nhc.gov.cn/xcs/xwbd/202002/35990d56cfcb43f4a7 0d7f9703b113c0.shtml. [Last accessed on 01 Jan 2021]

85. World Health Organization. Novel Coronavirus (2019-nCoV) situation report-7; 2020. Available from: https://www.who.int/docs/defaultsource/coronaviruse/situation-reports/20200127-sitrep-7-2019ncov.pdf?sfvrsn=98ef79f5_2. [Last accessed on 01 Jan 2021]

86. Centres for Disease Control and Prevention. Symptoms of Coronavirus. Centres for Disease Control and Prevention; 2020 Available from: https://www.cdc.gov/coronavirus/2019- 
ncov/symptoms-

testing/symptoms.html?CDC_AA_refVal=https $\% 3 \mathrm{~A} \% 2 \mathrm{~F} \% 2 \mathrm{Fwww}$. cdc. gov\%2Fcoronavirus\%2F2019-ncov\%2Fabout\%2 Fsymptoms. html. [Last accessed on 01 Jan 2021]

87. Sun K, Chen J, Viboud C. Early epidemiological analysis of the coronavirus disease 2019 outbreak based on crowdsourced data: a population-level observational study. Lancet 2020;2:201-8.

88. Worldometer. Coronavirus Incubation Period. Worldometer; 2020. Available

https://www.worldometers.info/coronavirus/coronavirusincubation-period/\#asy. [Last accessed on 01 Jan 2021]

89. Backer JA, Klinkenberg D, Wallinga J. Incubation period of 2019 novel coronavirus (2019-nCoV) infections among travellers from Wuhan, China, 20-28 January 2020. Euro Surveill 2020;25:5.

90. Reuters. Coronavirus incubation could be as long as $27 \mathrm{~d}$, Chinese provincial government says. Reuters; 2020. Available from: https://www.reuters.com/article/us-china-healthincubation/coronavirus-incubation-could-be-as-long-as-27days-chinese-provincial-government-says-idUSKCN20G06W. [Last accessed on 01 Jan 2021]

91. Bai Y, Yao L, TaoWei, Tian F, Jin DY, Chen L, et al. Presumed asymptomatic carrier transmission of COVID-19. Am Med Assoc 2020;323:1406-7.

92. World Health Organization. Coronavirus disease 2019 (COVID19) situation report-75. Vol. 2019; 2020.

93. World Health Organization. Coronavirus disease 2019 (COVID19) situation report-88. Vol. 2019; 2020.

94. World Health Organization. Safe Ramadan practices in the context of the COVID-19. 2020. Available from: https://www.who.int/publications-detail/safe-ramadanpractices-in-the-context-of-the-covid-19-interim-guidance. [Last accessed on 01 Jan 2021]

95. World Health Organization. Coronavirus disease 2019 (COVID19) situation report-100. Vol. 2019; 2020.

96. Worldometer. COVID-19 coronavirus pandemic. Worldometer; 2020.

97. Worldometer. COVID-19 coronavirus pandemic. Worldometer; 2021. Available from: https://www.worldometers.info/coronavirus/. [Last accessed on 01 Jan 2021]

98. Worldometer. COVID-19 coronavirus pandemic. Worldometer; 2020. p. 1-22. Available from: https://www.worldometers.info/coronavirus/\#news. [Last accessed on 21 Apr 2020]

99. World Health Organization. Coronavirus disease (COVID-19) situation report-102; 2020. Available from: https://www.who.int/docs/defaultsource/coronaviruse/situation-reports/20200501-covid-19sitrep.pdf?sfvrsn=742f4a18_2. [Last accessed on 01 Jan 2021]

100. Ghosh P, Ghosh R, Chakraborty B. COVID-19 in India: statewise analysis and prediction. JMIR Public Heal Surveill 2020;6:e20341-e20341.

101. PRS Legislative Researh. COVID-19 State wise data: India; 2021. p. 1-3. Available from: https://prsindia.org/covid-19/cases. [Last accessed on 01 Jan 2021]

102. Hindustan Times. Coronavirus: 4 northeast states untouched by Covid-19, no cases in Lakshadweep. Hindustan Times; 2020. p. 1-12. Available from: https://www. hindustantimes. com/india-news/coronavirus-4-northeast-states-untouchedby-covid-19-no-cases-in-lakshadweep/storycb7jo3clLkGFJeMYPFpvGL.html. [Last accessed on 01 Jan 2021]

103. The Times of India. Covid-19: Govt bans all international flights to India from March 22 to 29 | India News-Times of India; 2020. p. 1-4. Available from: https://timesofindia.indiatimes.com/india/govt-bans-allinternational-flights-to-india-from-march-22-to29/articleshow/74720923.cms. [Last accessed on 01 Jan 2021]

104. My Gov Cell. COVID19 statewise status. National Informatics Centre (NIC); 2021. p. 1-2. Available from: https://www.mygov.in/corona-data/covid19-statewisestatus/. [Last accessed on 01 Jan 2021]

105. World Health Organization. Emergencies preparedness, response: SARS-CoV-2 Variants; 2020. Available from: https://www.who.int/csr/don/31-december-2020-sars-cov2variants/en/ [Last accessed on 01 Jan 2021]

106. World Health Organization. COVID-19: WHO European Region Operational Update Vol. 53; 2021. Available from: https://www.euro.who.int/_data/assets/pdf_file/0011/4793 96/WHO-Regional-Office-for-Europe-Operational-UpdateWeeks-51-53-eng.pdf. [Last accessed on 01 Jan 2021]

107. European Centre for Disease Prevention and Control (ECDC). Coronavirus disease 2019 (COVID-19) in the EU/EEA and the UK-eighth update. Vol. 2019; 2020. Available from: https://www.ecdc.europa.eu/sites/default/files/documents/c ovid-19-rapid-risk-assessment-coronavirus-disease-2019eighth-update-8-april-2020.pdf. [Last accessed on 01 Jan 2021]

108. Worldometer. Coronavirus Cases Statistics and Charts. Worldometer. 2020[cited 2020 May 10]. Available from: https://www. worldometers. info/coronavirus/coronaviruscases/. [Last accessed on 01 Jan 2021]

109. Worldometer. Coronavirus Graphs: Worldwide Cases and Deaths-Worldometer. Worldometer; 2021. p. 1-29. Available from:

https://www.worldometers.info/coronavirus/worldwidegraphs/. [Last accessed on 01 Jan 2021]

110. Worldometer. COVID-19 coronavirus pandemic. Worldometer; 2020. Available from: https://www.worldometers.info/coronavirus/. [Last accessed on 10 May 2020]

111. Worldometer. Coronavirus Cases: Statistics and ChartsWorldometer. Vol. January 27, Worldometer; 2021. p. 1-12. Available from: https://www.worldometers. info/coronavirus/ coronavirus-cases/. [Last accessed on 01 Jan 2021]

112. Craven J. COVID-19 Vaccine Tracker. Regulatory Affairs Professionals Society (RAPS); 2021. p. 1-20. Available from: https://www.raps.org/news-and-articles/newsarticles/2020/3/covid-19-vaccine-tracker. [Last accessed on 01 Jan 2021]

113. World Health Organization. Access and allocation: how will there be fair and equitable allocation of limited supplies? World Health Organization; 2021. p. 1-6. Available from: https://www.who.int/news-room/featurestories/detail/access-and-allocation-how-will-there-be-fairand-equitable-allocation-of-limited-supplies. [Last accessed on 01 Jan 2021]

114. World Health Organization. The different types of COVID-19 vaccines. World Health Organization; 2021. p. 1-6. Available from: https://www.who.int/news-room/featurestories/detail/the-race-for-a-covid-19-vaccine-

explained.access and allocation: how will there be fair and equitable allocation of limited supplies. [Last accessed on 01 Jan 2021]

115. Nair KS, Kamath S, Rajan A, Thomas S, Aswin D, Zachariah SM Detailed view on repurposed drugs, tracking of vaccines,and brief view on prophylactic nanomedicines as an alternative approach and patient care for covid-19. Int J Appl Pharm 2021;13:19-26.

116. Adnan N, Nordin SM, Anwar A. Transition pathways for Malaysian paddy farmers to sustainable agricultural practices: an integrated exhibiting tactics to adopt Green fertilizer. Land use policy. 2020;90:104255. Available from: https://doi.org/10.1016/j.landusepol.2019.104255. [Last accessed on 01 Jan 2021]

117. Elmi A, Sayem SAJ, Ahmed M, Mohamed F. Natural compounds from djiboutian medicinal plants as inhibitors of COVID-19 by in silico investigations. Int J Curr Pharm Res 2020;12:4.

118. University of Oxford. Oxford COVID-19 vaccine begins human trial stage. Oxford University Medical Sciences Division; 2020. Available from: https://www.ovg.ox.ac.uk/news/oxford-covid19-vaccine-begins-human-trial-stage. [Last accessed on 01 Jan 2021]

119. UPMC. COVID-19 Vaccine Candidate Shows Promise. UPMC; 2020. Available from: https://www.upmc.com/media/news/040220-falo-gambottosars-cov2-vaccine. [Last accessed on 01 Jan 2021]

120. Novavax I. Novavax Identifies Coronavirus Vaccine Candidate; 2020. Available from: http://ir.novavax.com/news- 
releases/news-release-details/novavax-identifies-coronavirusvaccine-candidate-accelerates\#. [Last accessed on 01 Jan 2021]

121. Miller A, Reandelar MJ, Fasciglione K, Roumenova V, Li Y, Otazu GH. Correlation between universal BCG vaccination policy and reduced morbidity and mortality for COVID-19: an epidemiological study. medRxiv; 2020.

122. U. S. National Library of Medicine. Reducing Health Care Workers Absenteeism in Covid-19 Pandemic Through BCG Vaccine (BCGCORONA). ClinicalTrials.gov; 2020. Available from: https://clinicaltrials.gov/ct2/show/study/NCT04328441. [Last accessed on 01 Jan 2021]

123. Zhang L, Liu Y. Potential Interventions for novel coronavirus in China: a systemic review. J Med Virol 2020;92:479-90.

124. Shrivastava A. Analytical methods for the determination of hydroxychloroquine in various matrices. Int J Appl Pharm 2020;12:55-61.

125. Cavanagh D. Severe acute respiratory syndrome vaccine development: experiences of vaccination against avian infectious bronchitis coronavirus. Avian Pathol 2003;32:567-82.

126. Bijlenga G. Proposal for vaccination against SARS coronavirus using avian infectious bronchitis virus strain $\mathrm{H}$ from the netherlands. J Infect 2005;51:263-5.
127. Marano G, Vaglio S, Pupella S, Facco G, Catalano L, Giancarlo M et al. Convalescent plasma: new evidence for an old therapeutic tool? Blood Transfus 2016;14:152-7.

128. Arabi Y, Balkhy H, Hajeer AH, Bouchama A, Hayden FG, Omari A $\mathrm{Al}$, Hameed FM Al, et al. Feasibility, safety, clinical, and laboratory effects of convalescent plasma therapy for patients with Middle East respiratory syndrome coronavirus infection: a study protocol. Springer Plus 2015;4:1-8.

129. Soo YOY, Cheng Y, Wong R, Hui DS, Lee CK, Tsang KKS, et al. Retrospective comparison of convalescent plasma with continuing high-dose methylprednisolone treatment in SARS patients. Eur Soc Clin Microbiol Infect Dis 2004;10:676-8.

130. Wong YCR, Wong YOYSWS, Ng CKLMHL, Wong PCKC, Cheng CBLG. Use of convalescent plasma therapy in SARS patients in Hong Kong. Eur J Clin Microbiol Infect Dis 2005;24:44-6.

131. Velthuis AJW, Worm SHE Van Den, Sims AC, Baric RS, Snijder EJ, Hemert MJ Van. Zn 2+Inhibits coronavirus and arterivirus rna polymerase activity in vitro and zinc ionophores block the replication of these viruses in cell culture. PLoS Pathog 2010;6:e1001176. 